ARTICLE

\title{
NBR1 is a critical step in the repression of thermogenesis of p62-deficient adipocytes through PPAR $\gamma$
}

Jianfeng Huang1, Juan F. Linares ${ }^{2,3}$, Angeles Duran²,3, Wenmin Xia4, Alan R. Saltiel (1) 4, Timo D. Müller 5,6,7, Maria T. Diaz-Meco (i] ${ }^{2,3}$ \& Jorge Moscat (i] ${ }^{2,3 \times}$

Activation of non-shivering thermogenesis is considered a promising approach to lower body weight in obesity. p62 deficiency in adipocytes reduces systemic energy expenditure but its role in sustaining mitochondrial function and thermogenesis remains unresolved. NBR1 shares a remarkable structural similarity with p62 and can interact with p62 through their respective PB1 domains. However, the physiological relevance of NBR1 in metabolism, as compared to that of p62, was not clear. Here we show that whole-body and adipocyte-specific ablation of NBR1 reverts the obesity phenotype induced by p62 deficiency by restoring global energy expenditure and thermogenesis in brown adipose tissue. Impaired adrenergic-induced browning of p62-deficient adipocytes is rescued by NBR1 inactivation, unveiling a negative role of NBR1 in thermogenesis under conditions of p62 loss. We demonstrate that upon p62 inactivation, NBR1 represses the activity of PPAR $\gamma$, establishing an unexplored p62/NBR1mediated paradigm in adipocyte thermogenesis that is critical for the control of obesity.

\footnotetext{
${ }^{1}$ Sanford Burnham Prebys Medical Discovery Institute, La Jolla, CA, USA. ${ }^{2}$ Department of Pathology and Laboratory Medicine, Weill Cornell Medicine, New York, NY, USA. ${ }^{3}$ Sandra and Edward Meyer Cancer Center, Weill Cornell Medicine, New York, NY, USA. ${ }^{4}$ Division of Metabolism and Endocrinology, Department of Medicine, University of California, San Diego, La Jolla, CA, USA. ${ }^{5}$ Institute for Diabetes and Obesity, Helmholtz Diabetes Center at Helmholtz Centre Munich, Oberschleißheim, Germany. ${ }^{6}$ German Center for Diabetes Research (DZD), Neuherberg, Germany. ${ }^{7}$ Department of Pharmacology and Experimental Therapy, Institute of Experimental and Clinical Pharmacology and Toxicology, Eberhard Karls University Hospitals and Clinics,

Tübingen, Germany. ${ }^{凶}$ email: jom4010@med.cornell.edu
} 
$\mathrm{T}$ here are at least three main morphologically and functionally different adipocyte types: white, brown, and beige. Unlike white adipocytes, which are specialized in the storage of chemical energy in the form of triglycerides, classical brown adipocytes (BAs) are found in the interscapular area (termed interscapular brown adipose tissue, iBAT) in rodents and generate heat during cold exposure by an adaptive mechanism called non-shivering thermogenesis ${ }^{1}$. This process requires the expression of the uncoupling protein 1 (UCP1) in the inner mitochondrial membrane to uncouple oxidative phosphorylation from ATP regeneration, thereby dissipating the energy from electron transport as heat ${ }^{2}$. Active BAT is detected in cervical, supraclavicular, paravertebral, and deep neck regions, and is acutely induced by cold exposure. Since the amount of metabolic active BAT inversely correlates with body mass index in adult humans $^{3-5}$, and BAT is believed to help reduce adiposity, the epidemic of obesity and diabetes has greatly increased the interest in this type of metabolically active type of fat ${ }^{6}$. A better understanding of BAT biology and physiology would greatly help in the identification of effective therapeutic targets for obesity and related metabolic diseases ${ }^{7}$.

p62 (encoded by Sqstm1) has been proposed as an autophagy adaptor required for the packing and delivery of polyubiquitinated, misfolded proteins, and dysfunctional organelles for their clearance through autophagy during basal detoxification or waste removal in response to metabolic stress ${ }^{8}$. However, it is also well recognized that the function of p62 extends beyond autophagy $^{9-12}$. p62 acts as a multifunctional signaling hub due to its ability to interact with different key signaling proteins through well-defined structural elements ${ }^{8}$. Such interactions account for p62's roles in pathways controlling inflammation, cell death, survival, and metabolism ${ }^{9,13}$. With respect to metabolism, our previous results showed that total body inactivation of p62 resulted in mature-onset obesity due to reduced energy expenditure $(\mathrm{EE})^{14,15}$. We also demonstrated that the selective inactivation of p62 in adipocytes, and also specifically in BAT, recapitulated the impaired $\mathrm{EE}$ and the obesity phenotype of total body knockout $(\mathrm{KO})$ mice $^{16-18}$. Therefore, adipocyte's p62 emerges as a critical regulator of energy balance and adiposity in vivo. NBR1 can also function as an autophagic adaptor with remarkable similarity in domain organization to $\mathrm{p} 62$, and has been suggested to form dimers with p62 through their respective PB1 domains ${ }^{13,19}$. We have previously reported that NBR1 inactivation in the mouse myeloid compartment impairs adipose tissue inflammation driven by M1 polarized macrophages, which results in improved glucose tolerance in obese mice ${ }^{20}$. Although these results demonstrated a role of NBR1 in the control of the consequences of increased adiposity, they did not address the potential intrinsic role of NBR1 in adipocyte biology.

In this work, we provide evidence supporting the concept that the nuclear interaction of p62 and NBR1 and its fine tuning of the activity of peroxisome proliferator-activated receptor $\gamma(\operatorname{PPAR} \gamma)$ in adipocytes is essential for the stimulation of the thermogenic program of BAT and activation of adaptive thermogenesis.

\section{Results}

Loss of NBR1 inhibits increased adiposity of Sqstm1-deficient mice. To address the role of NBR1 in adipocyte biology, we generated a total body NBR1 KO $\left(\mathrm{Nbr1}^{-/-}\right)$mouse line and compared its phenotype with that of total body p62 $\mathrm{KO}\left(\mathrm{Sqstm1}^{-/-}\right)$. Interestingly, and as previously reported ${ }^{14}, S q s t m 1^{-/-}$mice had more body weight than wild-type (WT) mice (Fig. 1a), which was associated with increased masses of both white adipose tissue (WAT) and BAT (Fig. $1 \mathrm{~b}-\mathrm{d}$ ). In contrast, $\mathrm{Nbr1}^{-/-}$mice displayed no such metabolic phenotype (Fig. 1a-d), suggesting that NBR1 does not play a relevant role in whole-body metabolism. However, the analysis of mice with total body KO of both p62 and NBR1 (Sqstm1 $1^{-1}$ $\left.\mathrm{Nbr1}^{-/-}\right)$revealed that the increased fat weight of Sqstm1 ${ }^{-/}$mice was restored to WT conditions in the absence of NBR1 (Fig. 1b-d). Consistently, while p62-deficient mice exhibited increased lipid accumulation in BAT and enlarged adipocyte size in epididymal WAT (eWAT), this phenotype was completely rescued in Sqstm $1^{-/-}$ $\mathrm{Nbr1}^{-{ }^{-}}$mice (Fig. le-g). Moreover, the mRNA levels of two master thermogenic regulators (Ucp1 and Pgc1 $\alpha$ ), which were reduced in Sqstm1 1/- BAT, were rescued in Sqstm1 1/- $\mathrm{Nbr1}^{-/-}$BAT (Fig. 1h). Furthermore, the expression of key lipogenic genes that were increased in the eWAT of Sqstm1 $1^{-1-}$ mice was partially reduced to WT levels in Sqstm1 1/- $\mathrm{Nbr}^{-/-}$mice (Fig. 1i), likely reflecting the BAT-driven metabolic improvement in the host. These results demonstrate that NBR1 is an obligate step in the obesity phenotype unleashed by p62 deficiency, likely through the repression of the adipocyte's thermogenic program.

Adipocyte's NBR1 is required for increased adiposity driven by p62 deficiency. To determine whether the effect of inactivating global NBR1 on obesity could be accounted for by its potential role in adipocytes, we next generated a mouse line with the adipocyte-specific deletion of NBR1 either in WT mice $\left(N b r 1^{\mathrm{AKO}}\right)$ or in mice in which p62 has been selectively inactivated in adipocytes both in WAT and BAT (Sqstm1 $\left.1^{\mathrm{AKO}}\right)$. These adipocyte-selective double $\mathrm{KO}$ mice $\left(\right.$ Sqstm $\left.^{\mathrm{AKO}}{ }^{\mathrm{Nbr}} 1^{\mathrm{AKO}}\right)$ demonstrated that the specific loss of NBR1 in p62-deficient adipocytes rescued the body weight gain of Sqstm $1^{\mathrm{AKO}}$ mice to levels close to those of the corresponding WT controls (Fig. 2a, b). That is, BAT and WAT masses and whole-body fat composition, which were increased in $S q s t m 1^{\mathrm{AKO}}$ mice, were largely normalized in Sqstm $1^{\mathrm{AKO}} \mathrm{Nbr1} 1^{\mathrm{AKO}}$ mice (Fig. 2c-e). Notably, the normalization of body weight and fat mass in $\operatorname{Sqstm} 1 \mathrm{AKO}^{\mathrm{A} O}$ $b r 1^{\mathrm{AKO}}$ mice is independent of age and sex (Supplementary Fig. 1a-c). Histological analyses showed that while Sqstm $1^{\mathrm{AKO}}$ mice have robustly increased adipocyte size and lipid content in BAT and eWAT, these alterations were completely abrogated in Sqstm $1^{\mathrm{AKO}} \mathrm{Nbr} 1^{\mathrm{AKO}}$ mice (Fig. 2f, g and Supplementary Fig. 1d). We next determined the development of fatty liver by Oil Red O (ORO) staining and quantification of hepatic lipid content. In concordance with changes in adiposity, the fatty liver phenotype of Sqstm $1^{\mathrm{AKO}}$ mice was normalized in Sqstm $1^{\mathrm{AKO}} \mathrm{Nbr} 1^{\mathrm{AKO}}$ mice (Fig. 2f, h). Chronic inflammation in WAT, characterized by severe macrophage infiltration, may result in systemic insulin resistance in obese diabetic animals ${ }^{21}$. We found that the number of crown-like structures (a hallmark of macrophage infiltration) and the expression of macrophage marker F4/80 (encoded by Adgre1 gene), which were markedly increased in Sqstm $1^{\mathrm{AKO}}$ mice, were normalized in Sqstm $1^{\mathrm{AKO}} \mathrm{Nbr} 1^{\mathrm{AKO}}$ mice (Supplementary Fig. 1e, f). These findings suggest a functional dependence on NBR1 for p62 disfunction in adipocytes as the mechanism underlying the whole-body obese phenotype of Sqstm $1^{\mathrm{AKO}}$ mice.

NBR1 inactivation in adipocytes restores glucose intolerance and insulin resistance in Sqstm $1^{\mathrm{AKO}}$ mice. We next determined the impact of NBR1 deficiency in systemic glucose tolerance and insulin sensitivity. To this end, we measured glucose tolerance in mature mice of all genotypes. Interestingly, while glucose intolerance were evident characteristics of obese Sqstm 1 AKO mice in GTT (Fig. 3a), this phenotype was not observed in $N b r 1^{\mathrm{AKO}}$ and Sqstm $1^{\mathrm{AKO}}$ Nbr1 $1^{\mathrm{AKO}}$ mice (Fig. 3b, c). Next, insulin sensitivity was determined in these mice in ITT experiments. While insulin 


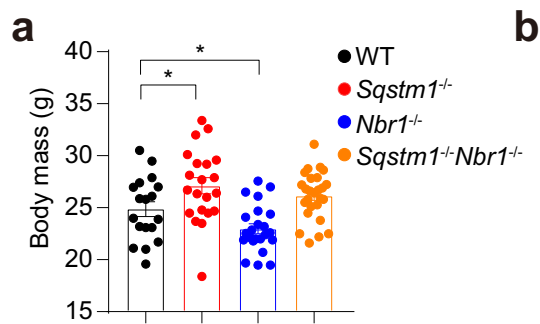

b

d

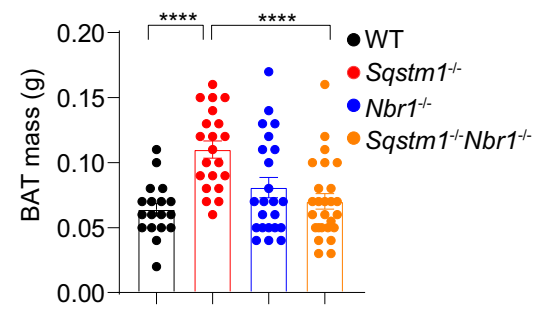

f

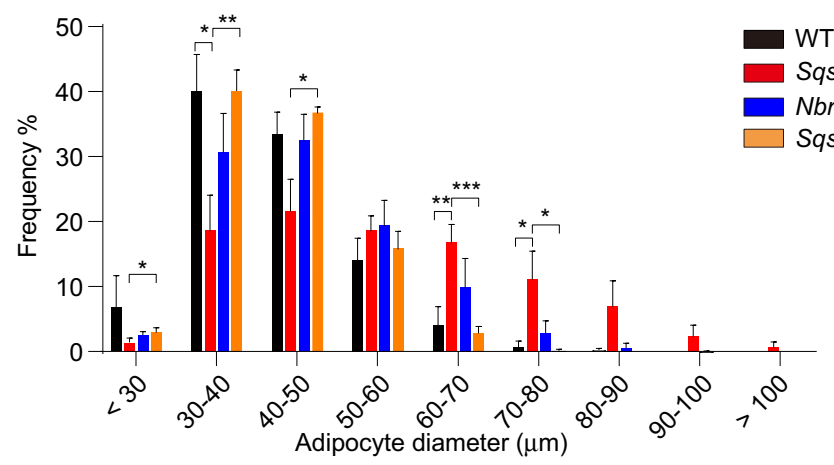

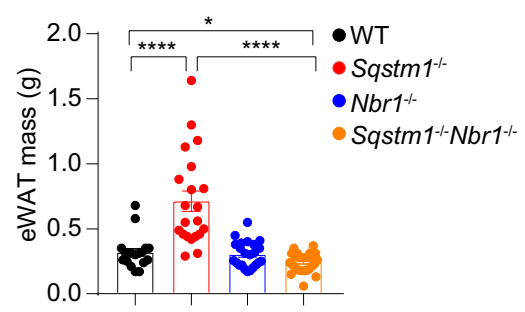

C

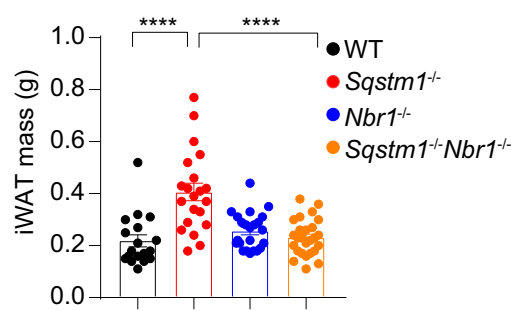

e

WT Sqstm 1 -
$\mathrm{Nbr1}^{-1-}$ qstm $1^{-1-N b r 1^{-1-}}$ h

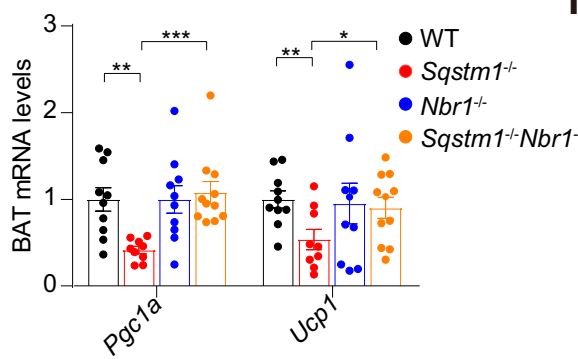

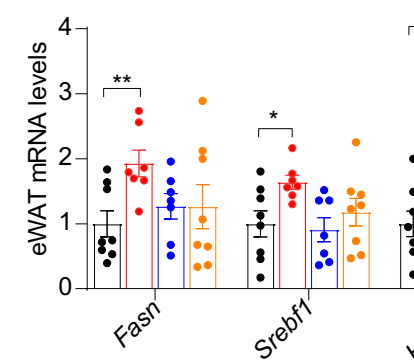

g

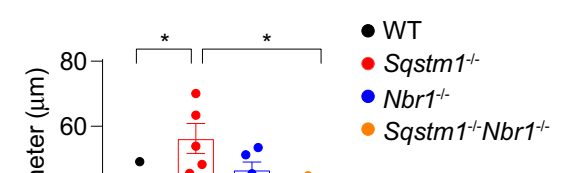

Fig. 1 Loss of NBR1 inhibits increased adiposity of Sqstm1-deficient mice. a-d Body mass (a) and fat tissue masses of eWAT (b), iWAT (c), and BAT (d)

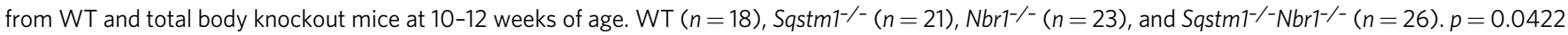

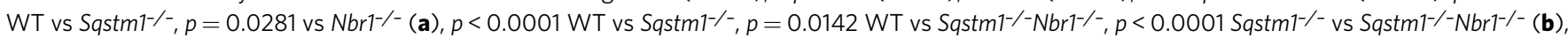

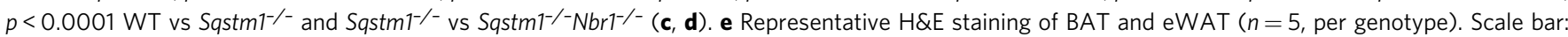
$100 \mu \mathrm{m}($ BAT) and $200 \mu \mathrm{m}$ (eWAT). f, g Adipocyte size measurement from H\&E staining of eWAT described above ( $n=5$ mice, per genotype).

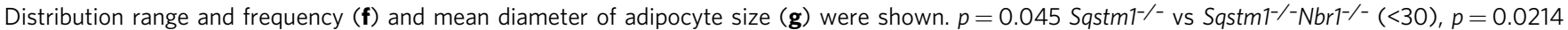

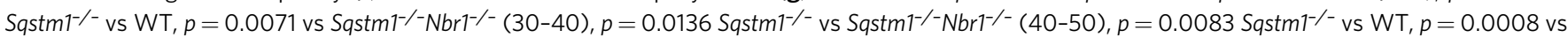

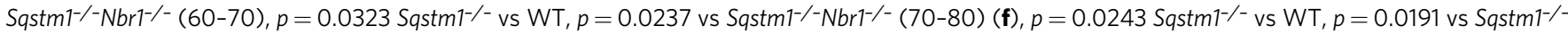
$\mathrm{Nbr1}^{-/-}(\mathbf{g})$. h qPCR analysis of thermogenesis genes in BAT. WT $(n=10), \mathrm{Sqstm}^{-/-}(n=9), \mathrm{Nbr1}^{-/-}(n=10)$, and Sqstm1 $1^{-/-} \mathrm{Nbr1^{-/- }}(n=11) . p=0.0011$

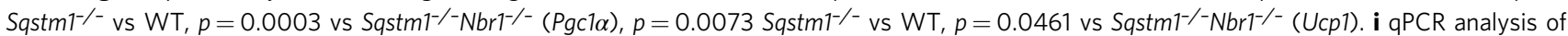

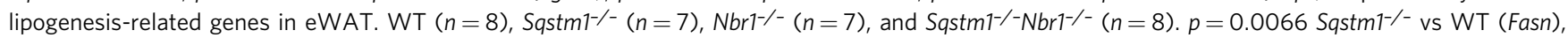

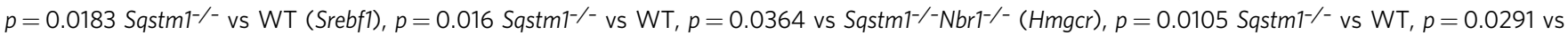

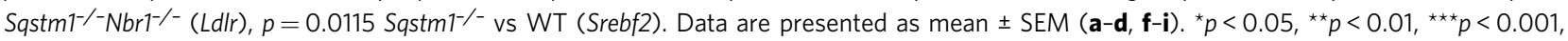
${ }^{\star \star \star \star} p<0.0001$. Unpaired two-tailed Student's $T$-test. Source data are provided as a Source Data file.

injection was insufficient to reduce the blood glucose concentrations in Sqstm $1^{\mathrm{AKO}}$ mice (Fig. 3d), Nbr1 ${ }^{\mathrm{AKO}}$ and Sqstm $1^{\mathrm{AKO}} \mathrm{Nbr} 1^{\mathrm{AKO}}$ mice exhibited similar responses to insulin as their flox controls (Fig. 3e, f). Although the initial glucose levels were higher in Sqstm $1^{\mathrm{AKO}} \mathrm{Nbr1} \mathrm{AKO}^{\mathrm{AK}}$ mice, likely due to unexpected fluctuation resulted from the short-term fasting procedure, presenting ITT data as percent of initial glucose levels clearly supports the notion that the Sqstm $1^{\mathrm{AKO}} N b r 1^{\mathrm{AKO}}$ mice manage to maintain insulin sensitivity but the Sqstm $1^{\mathrm{AKO}}$ mice fail to do so (Supplementary Fig. 2). Collectively, these results 

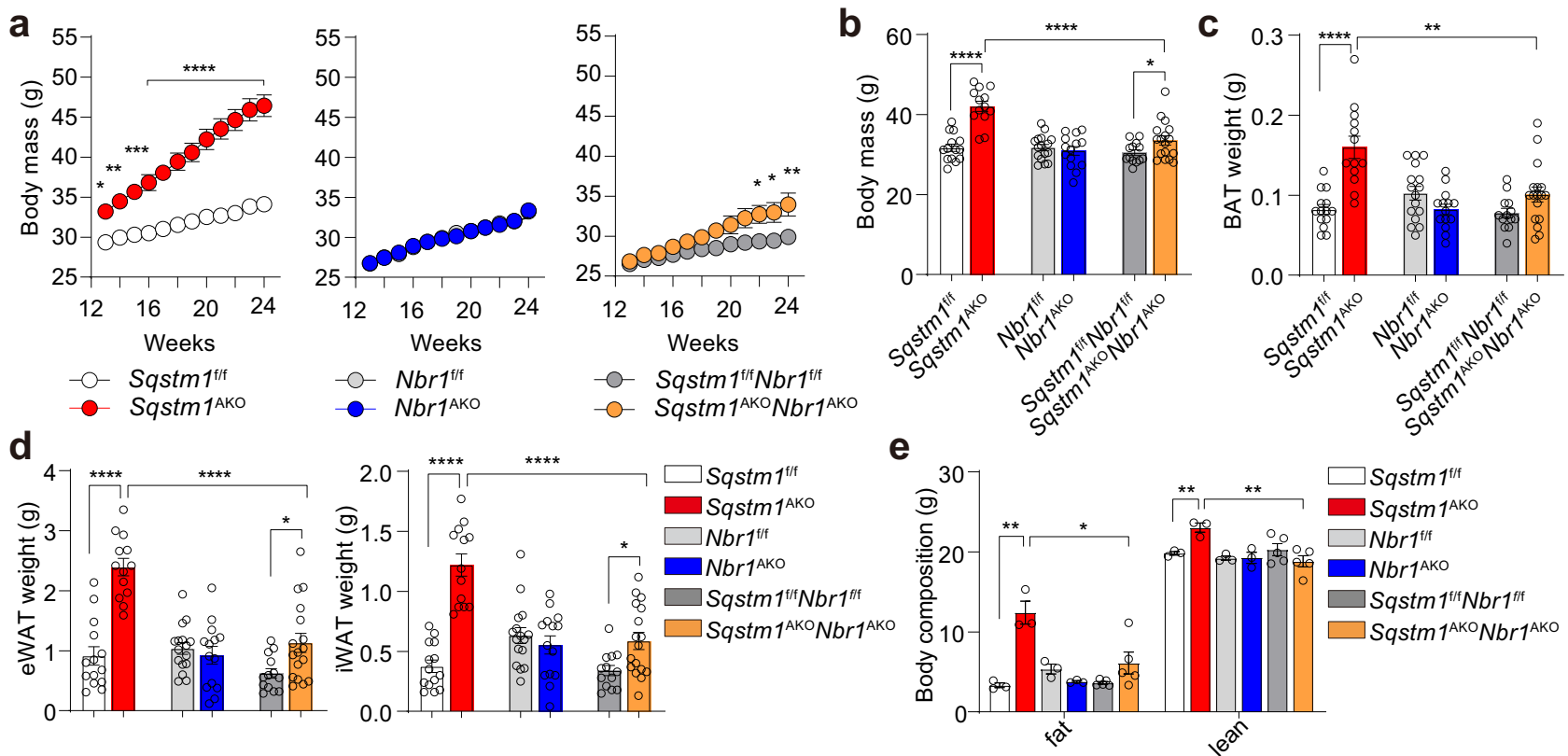

f

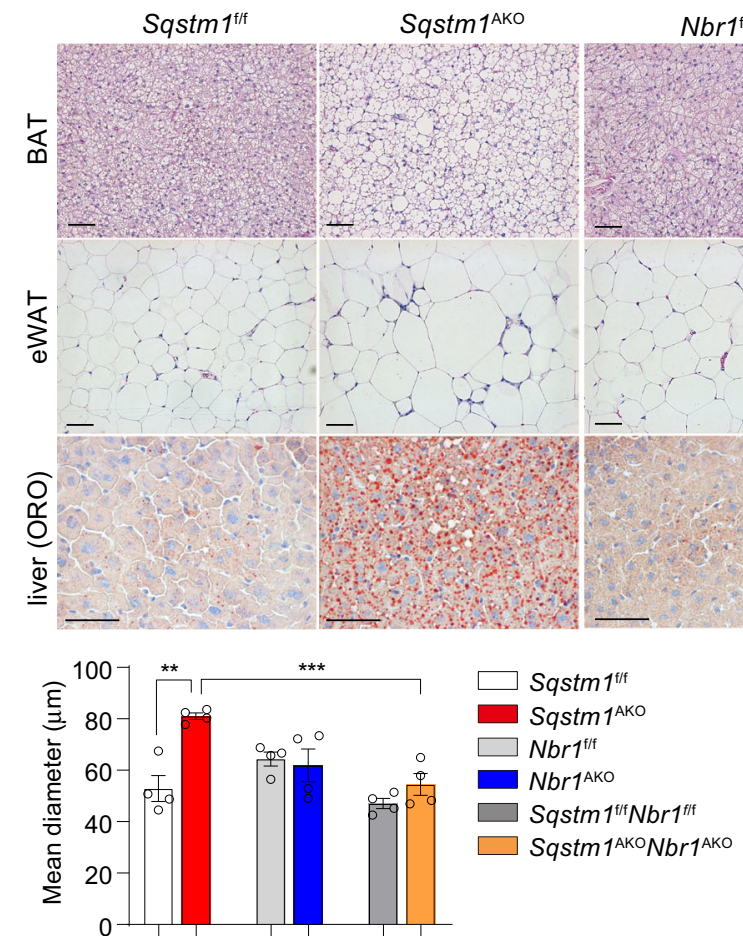

established that the loss of NBR1 in adipocytes protects mice from the dysfunctional glucose metabolism characteristic of adipocyte-specific p62 deficiency.

NBR1 inactivation in adipocytes restores impaired systemic energy expenditure in Sqstm $1^{\text {AKO }}$ mice. To evaluate the role of adipocyte NBR1 in whole-body metabolic profile, especially in the context of p62 deficiency, we performed a full metabolic characterization of the adipocyte-selective $\mathrm{KO}$ mouse lines. In concordance with the previous study ${ }^{16}$, p62 ablation in adipocytes led to marked reduction in EE as determined by ANCOVA using body weight as covariate as previously described ${ }^{22}$ (Fig. 4a, b). Interestingly, the reduced EE of Sqstm $1 \mathrm{AKO}$ mice was restored in Sqstm $1^{\mathrm{AKO}}$ Nbr $1^{\mathrm{AKO}}$ mice to WT levels (Fig. 4a, b). The respiratory exchange ratio (RER) denotes the preference for carbohydrates and lipids as fuels to fit the energy demand. The fact that Sqstm1 $\mathrm{AKO}$ mice had lower RER during the dark (feeding) phase suggests that most of the dietary carbohydrates are stored rather than being metabolized (Fig. 4c, d). Notably, this parameter was largely restored in Sqstm $1^{\mathrm{AKO}} \mathrm{Nbr} 1^{\mathrm{AKO}}$ mice when comparing to Sqstm $1^{\mathrm{AKO}}$ mice (Fig. 4c, d). This observation is consistent with an improvement in the whole-body metabolic rate, with no alterations in food intake and locomotor activity (Supplementary Fig. 3a, b). Furthermore, in agreement with their unaltered body weight and fat mass, $N b r 1^{\mathrm{AKO}}$ mice showed no phenotypic changes in any of the metabolic parameters investigated, including EE, RER, food intake, and locomotor activity (Fig. 4a-d and Supplementary Fig. 3a, b). These data support the notion that the loss of NBR1 in adipocytes rescues the impaired systemic EE driven by p62 deficiency. 
Fig. 2 Role of adipocyte NBR1 in the control of adiposity. a Recording body weight of adipocyte-specific knockout male mice and WT controls from 13 to

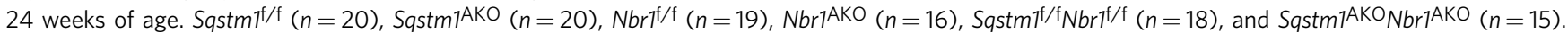
$p=0.0261(13 \mathrm{w}), p=0.0041(14 \mathrm{w}), p=0.0003(15 \mathrm{w}), p<0.0001(16-24 \mathrm{w})$ Sqstm7AKO vs Sqstmif/f $, p=0.0177(22 \mathrm{w}), p=0.012(23 \mathrm{w}), p=0.0018$ (24 w) Sqstm7 AKO NbriAKO vs Sqstm $7^{f / f} \mathrm{Nbr} 7^{f / f}$. b-d Body mass (b) and fat tissue weight of BAT (c) and eWAT, iWAT (d) from adipocyte-specific knockout

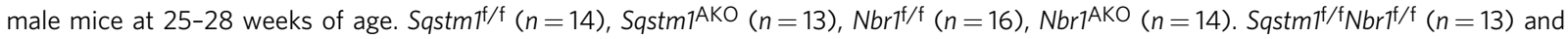

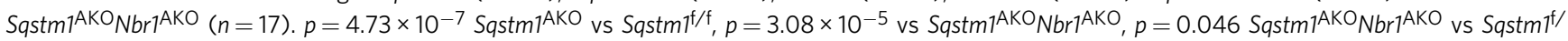

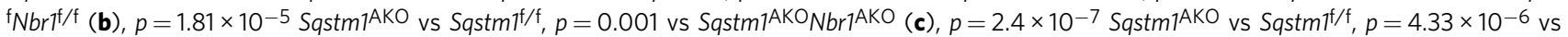

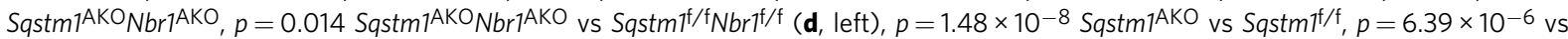

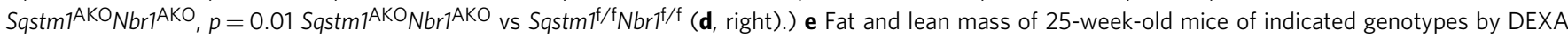

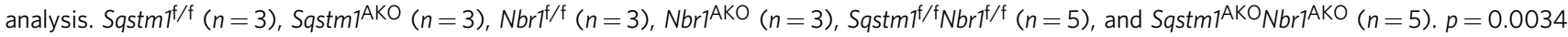

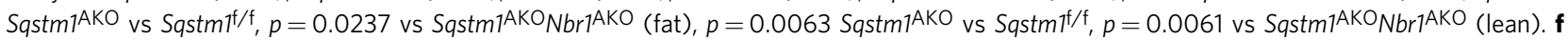
Representative H\&E staining in BAT and eWAT and Oil Red O (ORO) staining in livers. H\&E staining $\left(n=3\right.$ for Sqstm $7^{f / f}, S q s t m 7^{A K O}, N b r 7^{f / f}$ and $N b r 7^{A K O}$ and $n=4$ for Sqstm $7^{\mathrm{f} / \mathrm{f}} \mathrm{Nbr} \mathrm{f}^{\mathrm{f} / \mathrm{f}}$ and Sqstm $\left.{ }^{\mathrm{AKO}}{ }^{\mathrm{Nbr}} \mathrm{AKO}^{\mathrm{AKO}}\right)$, ORO staining $(n=3$, per genotypes). Scale bar $=100 \mu \mathrm{m}$. $\mathbf{g}$ Adipocyte size measurement from H\&E staining of eWAT described above $\left(n=4\right.$, per genotype). $p=0.0016$ Sqstm $^{\text {AKO }}$ vs Sqstm $7^{f / f}, p=0.00099$ vs Sqstm7 ${ }^{\text {AKO }}$ Nbri $^{\text {AKO }}$. h TG content

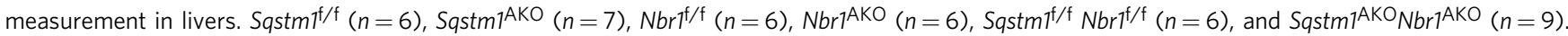
$p=0.0038 \mathrm{Sqstm} 7 \mathrm{AKO}$ vs Sqstm7 $/ \mathrm{f}, p=0.0001$ vs Sqstm7AKONbr7AKO. Data are presented as mean \pm SEM $(\mathbf{a}-\mathbf{e}, \mathbf{g}, \mathbf{h}) .{ }^{\star} p<0.05,{ }^{\star \star} p<0.01,{ }^{\star \star \star} p<0.001$, ${ }^{\star \star \star \star} p<0.0001$. Two-way ANOVA followed by Bonferroni's post-test (a). Two-tailed Student's $T$-test (b-h). Source data are provided as a Source Data file.
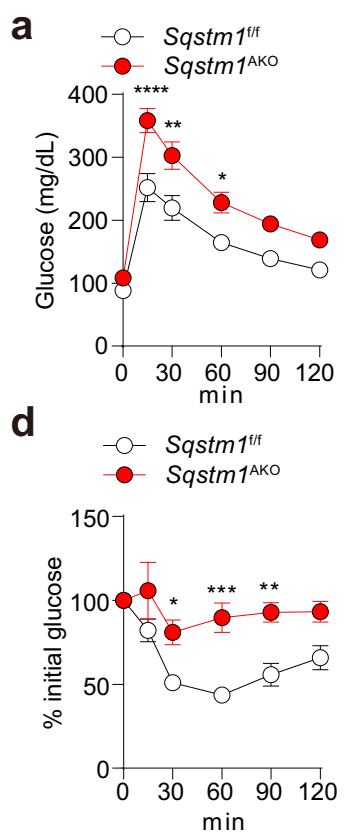

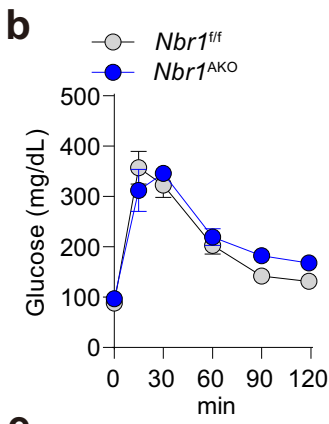

e

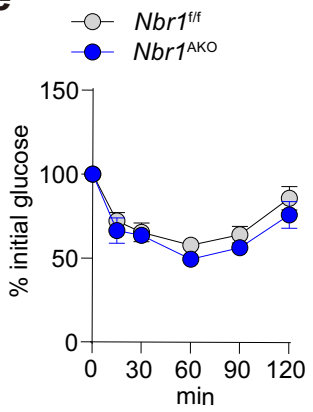

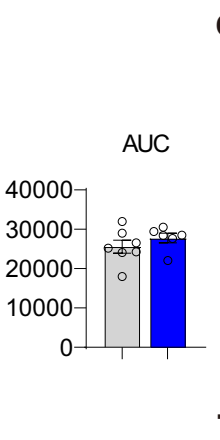
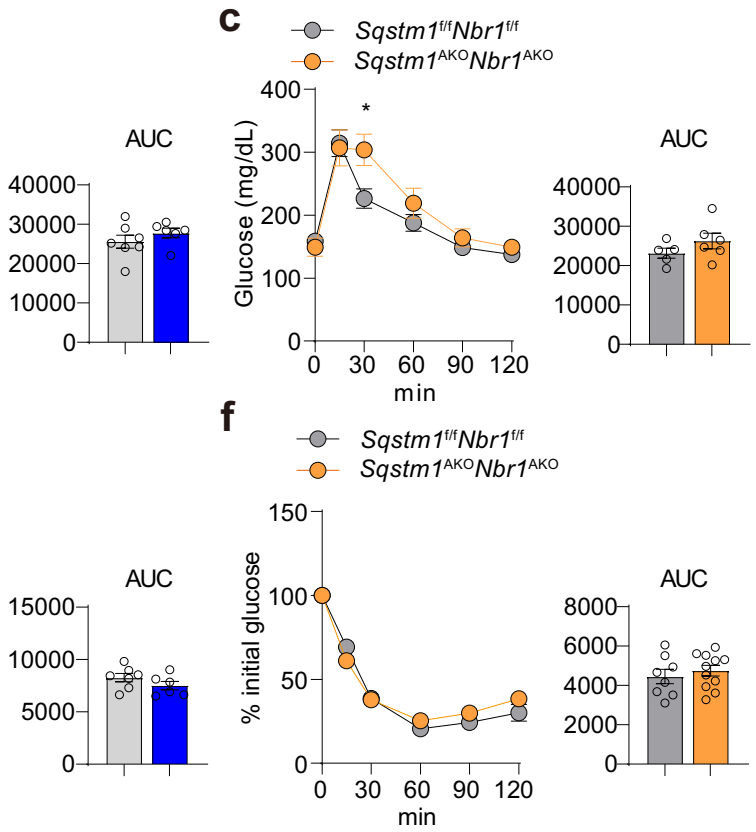

Fig. 3 Role of adipocyte NBR1 in the control of glucose intolerance and insulin resistance. a-c Glucose tolerance test (GTT) were performed in

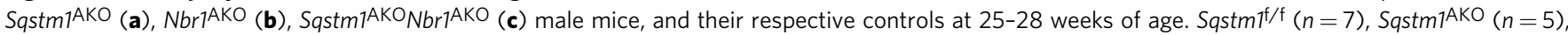
$\mathrm{Nbr}^{f / f}(n=7), \operatorname{Nbr} 7^{\mathrm{AKO}}(n=6), \mathrm{Sqstm}^{\mathrm{f} / \mathrm{f}} \mathrm{Nbr} 7^{\mathrm{f} / \mathrm{f}}(n=5)$, and Sqstm7 AKONbr7AKO $(n=6)$. Longitudinal graph: $p<0.0001, p=0.002, p=0.0319(\mathbf{a})$, $p=0.0318$ (c). Area under curves (AUC) were calculated from GTT. $p=0.0033$ (a). d-f Insulin tolerance test (ITT) was performed in Sqstm7 ${ }^{\mathrm{AKO}}$ (d), $\mathrm{Nbri}^{\mathrm{AKO}}(\mathbf{e}), \mathrm{S} q s t m^{\mathrm{AKO}} \mathrm{Nbr7}^{\mathrm{AKO}}(\mathbf{f})$ male mice and their respective controls at 25-28 weeks of age. Results are presented as percent of the initial glucose levels. Sqstm ${ }^{f / f}(n=7)$, Sqstm7 Ako $(n=6), \mathrm{Nbr}^{\mathrm{f} / \mathrm{f}}(n=7), \mathrm{Nbrl}^{\mathrm{AKO}}(n=6), \mathrm{Sqstm}^{\mathrm{f} / \mathrm{f}} \mathrm{Nbr} \mathrm{f}^{\mathrm{f} / \mathrm{f}}(n=8)$, and Sqstm7 AKO $\mathrm{Nbr}^{\mathrm{AKO}}(n=11)$. Longitudinal graph: $p=0.0273, p=0.0002, p=0.0035(\mathbf{d})$. AUC was calculated from ITT. Data are presented as mean \pm SEM $(\mathbf{a}-\mathbf{f}) .{ }^{\star} p<0.05,{ }^{\star \star} p<0.01,{ }^{\star \star \star} p<0.001$,

${ }^{\star \star \star} p<0.0001$. Two-way ANOVA followed by Bonferroni's post-test (a-f longitudinal graphs). Two-tailed Student's T-test (a-f AUC bar graphs). Source data are provided as a Source Data file.

Adipocyte NBR1 is required for downregulation of adaptive thermogenesis in BAT and inguinal WAT of p62-deficient mice. We next tested in these mice the adaptive thermogenic capacity of BAT. To this end, mice of the different genotypes were exposed to cold $\left(4^{\circ} \mathrm{C}\right)$ for $7 \mathrm{~h}$ to stimulate their thermogenic program. In contrast to the hypothermic Sqstm $1^{\mathrm{AKO}}$ mice, the Sqstm $1^{\mathrm{AKO}} N b r 1^{\mathrm{AKO}}$ and $N b r 1^{\mathrm{AKO}}$ mice were able to maintain their core temperature against acute cold exposure to levels similar to their respective WT controls, suggesting intact heat generation (Fig. 5a and Supplementary Fig. 4a). Interestingly, while a "whitening" histological feature was found in Sqstm $1^{\mathrm{AKO}}$ BAT upon cold exposure, indicative of insufficient lipid mobilization and metabolization, this abnormality was largely rescued in Sqstm $1^{\mathrm{AKO}} N b r 1^{\mathrm{AKO}}$ mice (Fig. 5b), while $N b r 1^{\mathrm{AKO}}$ mice showed no phenotype (Supplementary Fig. 4b). Consistently, the expression of thermogenic genes (Ucp1, Dio2, Cided, and $\operatorname{Cox} 7 \alpha)$ in response to cold-driven sympathetic stimulation was significantly decreased in Sqstm $1^{\text {AKO }}$ BAT but was largely rescued in Sqstm $1^{\mathrm{AKO}}{ }_{\mathrm{Nbr}}{ }^{\mathrm{AKO}}$ BAT (Fig. 5c).

Beige adipocytes are the inducible form of thermogenic fat cells that emerge within inguinal WAT in rodents in response to a variety of external stimuli, such as chronic cold exposure and cancer cachexia 17,23 . Injection of the $\beta_{3}$-adrenergic agonist $\mathrm{CL} 316,243$ in Sqstmi ${ }^{\mathrm{AKO}} \mathrm{Nbr1}^{\mathrm{AKO}}$ and $N b r 1^{\mathrm{AKO}}$ mice rapidly 
a

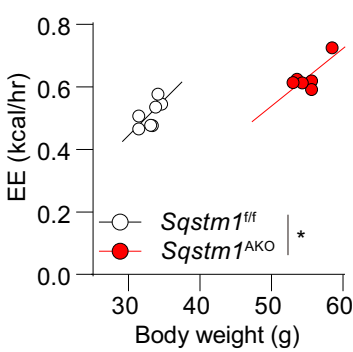

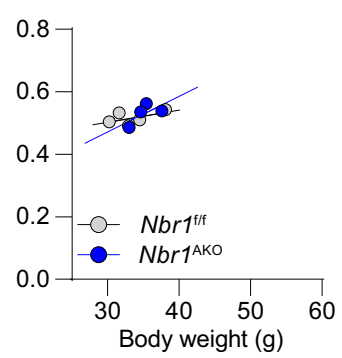

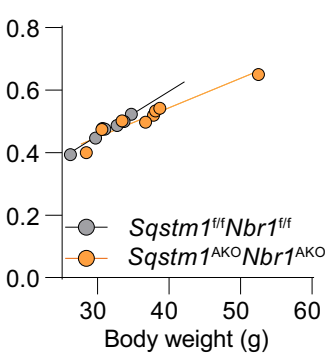

b

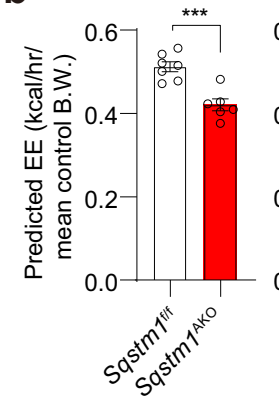

d
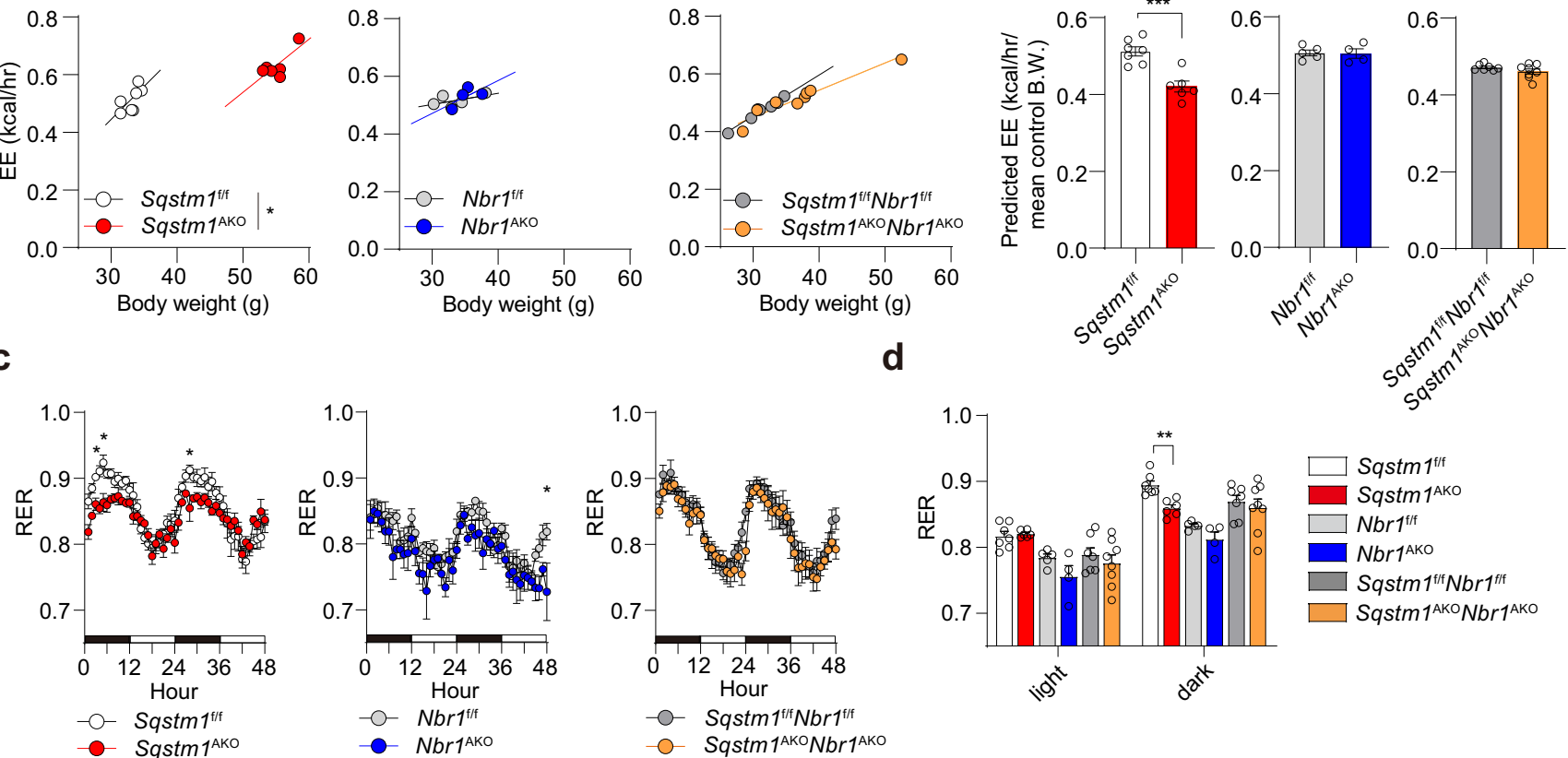

Fig. 4 Role of NBR1 in systemic energy expenditure. A metabolic characterization of the adipocyte-selective KO mice was performed by using an automated indirect calorimetry system (CLAMS) in male mice at 50-55 weeks of age. Sastm7 $7^{7 / \mathrm{f}}(n=7), \operatorname{Sastm} 7^{A K O}(n=6), \mathrm{Nbr} \mathrm{f}^{f / \mathrm{f}}(n=5), \mathrm{Nbr} \mathrm{f}^{\mathrm{AKO}}$

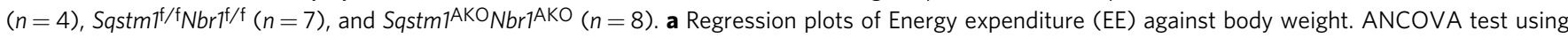
body weight as covariate. $p=0.043 \mathrm{Sqstm} 7^{\mathrm{AKO}}$ vs Sqstm $\mathrm{T}^{\mathrm{f} / \mathrm{f}}$. . Predicted energy expenditure at the mean body weight of respective flox control mice. The

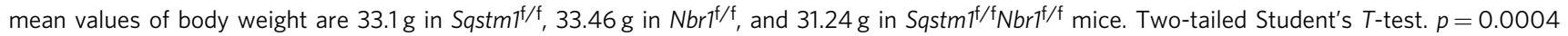
Sastm ${ }^{A K O}$ vs Sastm $7^{f / f}$. BW body weight. c Respiratory exchange rate (RER) was recorded and plotted. Two-way ANOVA followed by Bonferroni's post-

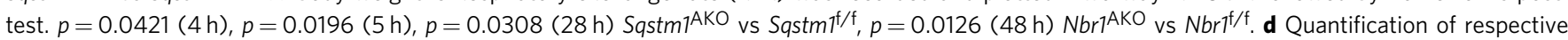
AUC from (c) was analyzed by two-tailed Student's $T$-test. $p=0.0012$ Sastm7AKO vs Sqstm $7^{f / f}$. Data are presented as mean \pm SEM (b-d). ${ }^{*} p<0.05$, ${ }^{\star \star} p<0.01,{ }^{\star \star \star} p<0.001$. Source data are provided as a Source Data file.

switched adipocytes from the characteristic unilocular to the multilocular cell morphology, which is typical of beige/bright cells (Fig. 5d and Supplementary Fig. 4c). However, inguinal white adipocytes in Sqstm $1^{\mathrm{AKO}}$ mice remained unilocular and enlarged in cell size (Fig. 5d), indicative of the absence of beige cells. The levels of UCP1 and mitochondrial complex proteins were upregulated by CL316,243 in Sqstm $1^{\mathrm{AKO}} N b r 1^{\mathrm{AKO}}$ mice, as well as that in WT controls whereas such an induction was largely diminished in Sqstm1 ${ }^{\mathrm{AKO}}$ mice (Fig. 5e-g). These results establish that p62 and NBR1 impact the thermogenesis program of both brown and beige adipocytes, and likely their mitochondrial function.

In this regard, our previously published results demonstrated that the loss of p62 in BAs resulted in impaired mitochondrial oxygen consumption rate (OCR) $)^{16}$. To determine the impact of NBR1 deficiency in this process, we first established primary BAs through differentiation of BAT stromal vascular fractions (SVF) isolated from either single or double $\mathrm{KO}$ mice. Under basal condition, Ucp1 mRNA levels were significantly decreased in primary Sqstm1 $1^{-/}$BAs, but that reduction was abrogated in Sqstm1 1-/- $\mathrm{Nbr1}^{-/-}$BAs (Fig. 6a). While increased UCP1 protein expression was induced by the adrenergic agonist isoproterenol (ISO) in all the four genotypes, the induction was comparably lower in primary Sqstm1 $1^{-/}$BAs than that in WT and Sqstm1 $1^{-/}$ $\mathrm{Nbr1}^{-/-}$BAs (Fig. 6b). We also generated immortalized brown adipocytes (iBAs). Interestingly, while the oligomycin-resistant mitochondrial OCR (basal uncoupling respiration) was decreased in Sqstm1 $1^{--}$iBAs, it remained unaltered in Sqstm1 $1^{-/} \mathrm{Nbr1}^{-/-}$ iBAs (Fig. 6c). Subsequent ISO injection elevated respiration levels (ISO-stimulated uncoupling OCR) in Sqstm1 1-/- $\mathrm{Nbr1}^{-/-}$ iBAs significantly higher than that in Sqstm1 $1^{-/}$iBAs (Fig. 6c).
BAT takes up large amounts of glucose during cold exposure in mice and humans. The cold-induced expression of glycolytic enzymes could be mimicked in vitro by $\beta$-adrenergic stimulation $^{24}$. We found that upon ISO stimulation, Sqstm $1^{-/-}$ $\mathrm{Nbr1}^{-/-}$iBAs exhibited higher ECAR relative to Sqstm1 $1^{-/-} \mathrm{iBAs}$ (Fig. 6d). These results demonstrated that the loss of NBR1 in BAs largely rescued the thermogenic and mitochondrial defects in Sqstm1 $1^{-/-}$BAT.

p62 and NBR1 interact with PPAR $\gamma$ in the nucleus of brown adipocytes. In search of the molecular crosstalk between p62 and NBR1 that might underly their role in adipocyte biology, we initially found that NBR1 expression was dramatically upregulated during brown adipogenesis, following a pattern similar to that of PPAR $\gamma$ expression (Supplementary Fig. 5a). Unlike NBR1, p62 showed just a marginal increase, detectable only in fully differentiated mature BAs (Supplementary Fig. 5a). Treatment with ISO that transcriptionally upregulates UCP1 and drives thermogenesis robustly induced the nuclear amounts of PPAR $\gamma$ and CREB, two master thermogenic regulators, but also triggered the nuclear translocation of p62 and NBR1 (Fig. 7a). We hypothesized that the impaired thermogenic and mitochondrial activity characteristic of p62 deficiency are due to a defective activation of the PPAR $\gamma$ transcriptional program. Since adrenergic stimulation augments the nuclear levels of p62, NBR1, and PPAR $\gamma$ (Fig. 7a), we speculated that they could be part of a multicomponent protein complex. Consistent with this hypothesis, PPAR $\gamma$ and NBR1 were co-immunoprecipitated with p62 in iBAs treated with ISO and rosiglitazone (Fig. 7b and Supplementary Fig. 5b). These observations demonstrate that both p62 and NBR1 can form a complex with PPAR $\gamma$. Because similar results 
a

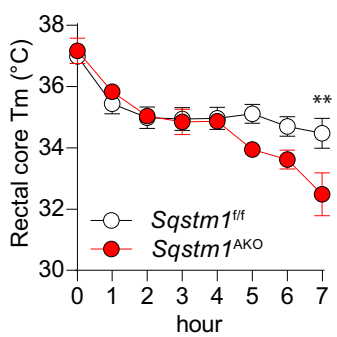

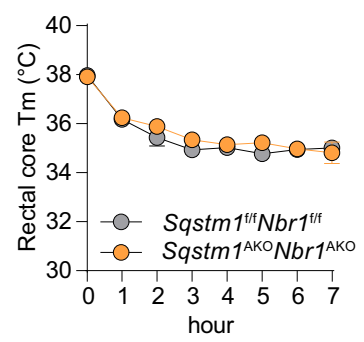

C
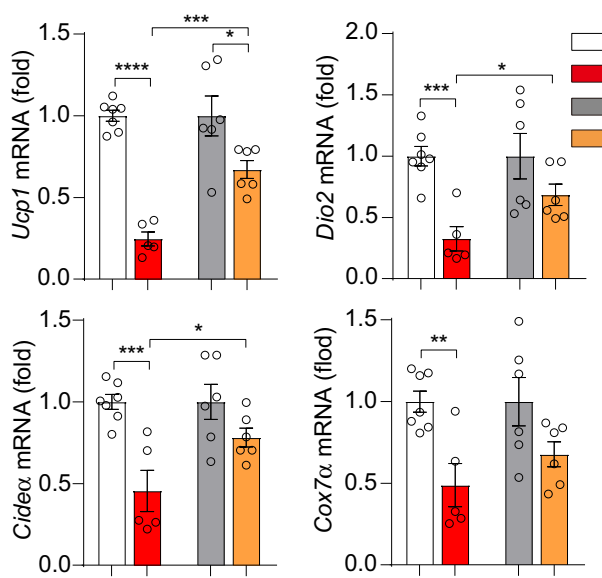

e

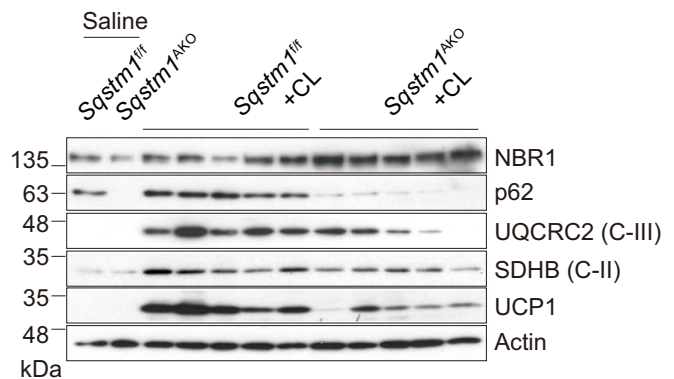

b

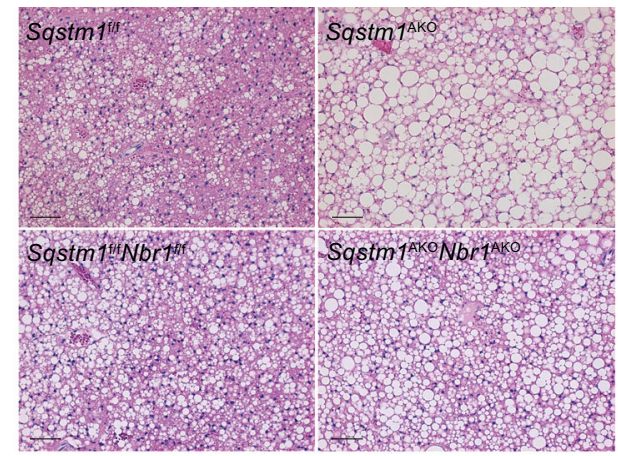

d

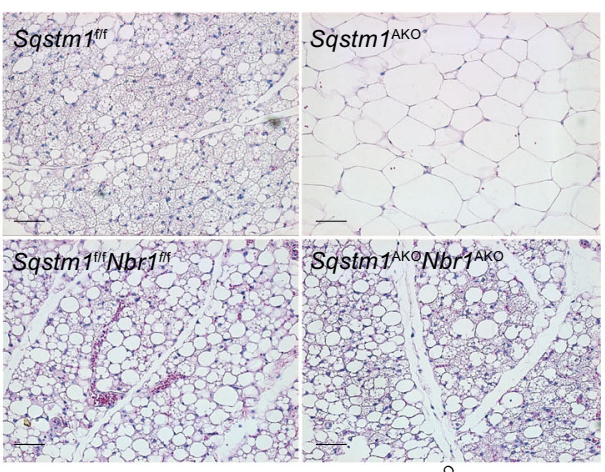

f

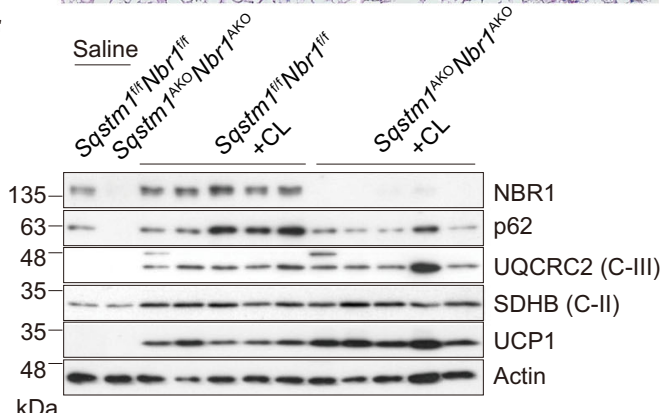

g

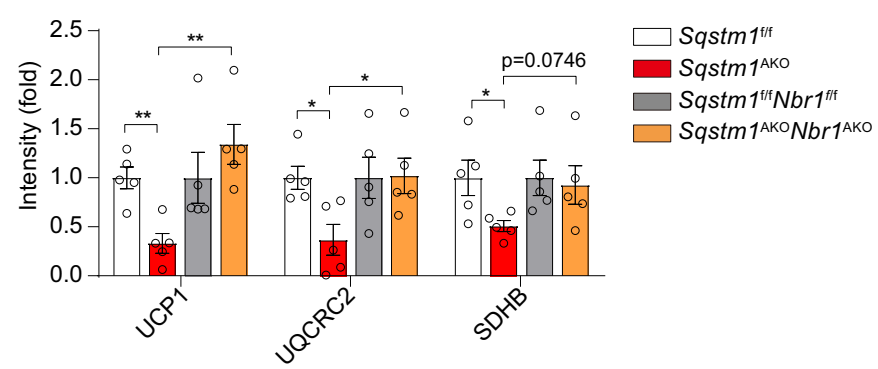

Fig. 5 Role of NBR1 in adaptive thermogenesis in BAT and inguinal WAT. a-c Male mice at 25 weeks of age were subjected to acute cold exposure $\left(4^{\circ} \mathrm{C}\right)$ for $7 \mathrm{~h}$ to stimulate brown thermogenesis. a Rectal core temperature was measured for consecutive $7 \mathrm{~h} . \mathrm{Sqstm} 7^{\mathrm{f} / \mathrm{f}}(n=10), \mathrm{Sqstm} 7^{\mathrm{AKO}}(n=6)$, Sqstmi $7^{f / f} \mathrm{Nbr}^{\mathrm{f} / \mathrm{f}}(n=9)$, and Sqstm7 AKO $\mathrm{Nbr}^{\mathrm{AKO}}(n=10)$. Two-way ANOVA followed by Bonferroni's post-test. b Representative H\&E staining in BAT of indicated mice $(n=3$, per genotype). Scale bar $=100 \mu \mathrm{m}$. c qPCR analysis of thermogenesis-related genes in BAT of mice. Results are presented as change fold related to individual controls. Sqstm $7^{\mathrm{f} / \mathrm{f}}(n=7)$, Sqstm $7^{\mathrm{AKO}}(n=5), \mathrm{Sqstm}^{\mathrm{f} / \mathrm{f}} \mathrm{Nbr}^{\mathrm{f} / \mathrm{f}}(n=6)$, and Sqstm7 ${ }^{\mathrm{AKO}} \mathrm{Nbr} 7^{\mathrm{AKO}}(n=6)$. Two-tailed Student's T-test. d-g Male mice at 25 weeks of age were injected with CL316,243 or saline as control for consecutive 5 days. d Representative H\&E staining in iWAT of

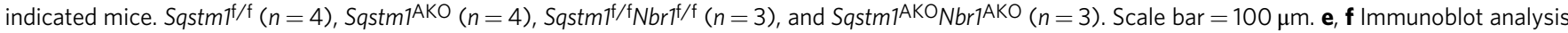
of mitochondrial OXPHOS genes and UCP1 in BAT of Sqstm7 AKO (e) and Sqstm7 ${ }^{A K O}$ Nbri $^{A K O}$ (f) and their respective controls ( $n=5$, per genotype).

$\mathbf{g}$ Densitometric quantification of gene intensity from western blot $(\mathbf{e}, \mathbf{f})$. Results are presented as change fold related to individual controls. Two-tailed Student's $T$-test. Data are presented as mean $\pm \operatorname{SEM}(\mathbf{a}, \mathbf{c}, \mathbf{g}) .{ }^{\star} p<0.05,{ }^{\star \star} p<0.01,{ }^{\star \star \star} p<0.001,{ }^{\star \star \star \star} p<0.0001$. Source data are provided as a Source Data file. 


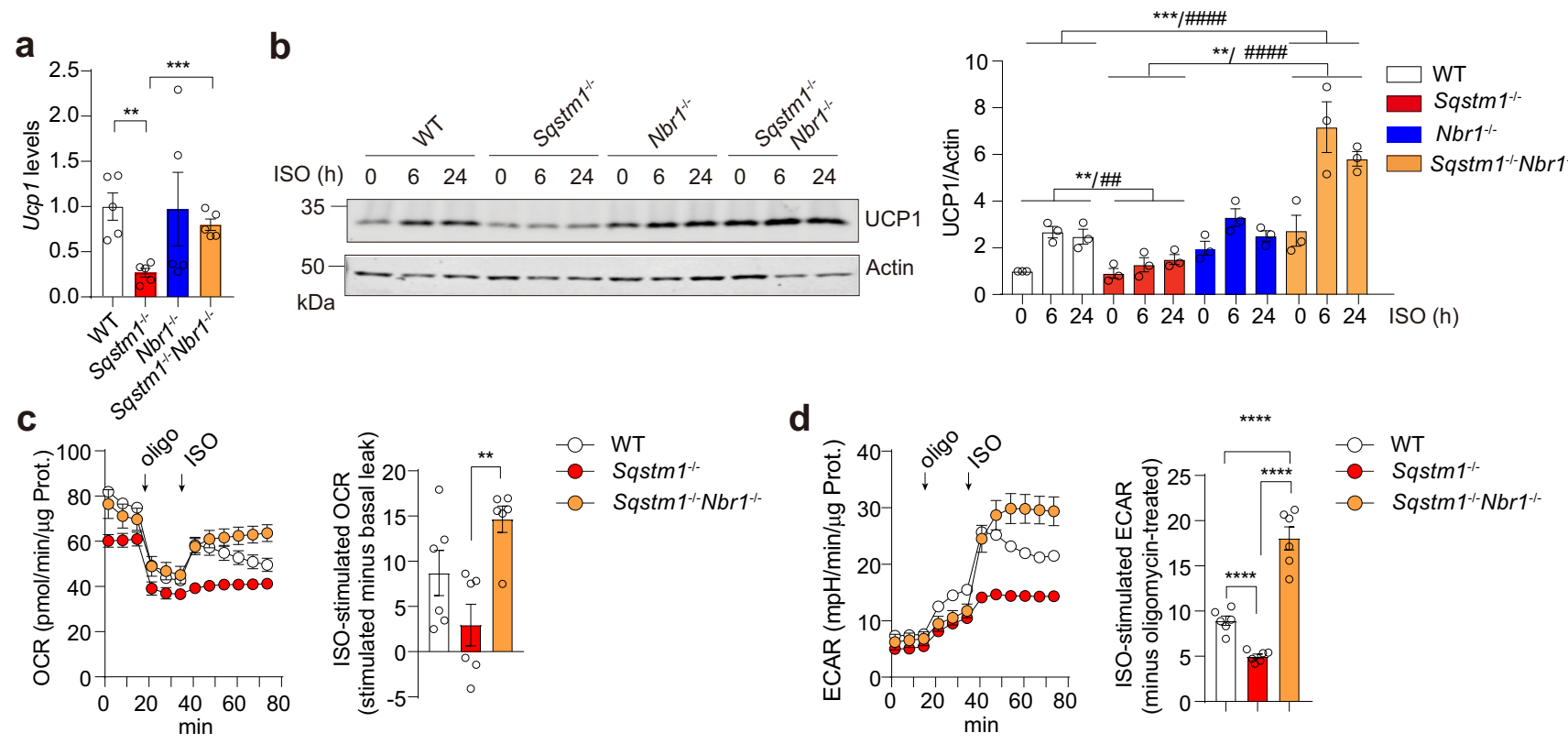

Fig. 6 Role of NBR1 in adaptive thermogenesis in primary brown adipocytes. a qPCR analysis of Ucp1 expression in primary brown adipocytes differentiated from SVF of four indicated genotypes ( $n=5$ biological replicates). b Immunoblot analysis of UCP1 in primary brown adipocytes of four indicated genotypes, treated with ISO $(0.5 \mu \mathrm{M})$ for indicated time. Representative blots (left) and quantification (right) from three independent experiments were shown. c, d Immortalized SVF from WT, Sqstm1 ${ }^{-/-}$, and Sqstm1 $1^{-/-} \mathrm{Nbr}^{-/-}$mice were differentiated into brown adipocytes for Seahorse analyses. c Oxygen consumption rate (OCR) were determined ( $n=6$ biological replicates). Left: plot of OCR to time measured by Seahorse. Right: calculated ISO-stimulated respiration levels after subtracting levels of basal proton leak. $\mathbf{d}$ Extracellular acidification rate $(E C A R)$ were measured $(n=6$ biological replicates). Left: plot of ECAR to time measured by Seahorse. Right: calculated ISO-stimulated ECAR values after subtracting oligomycin-treated levels. Data are presented as mean \pm SEM (a-d). ${ }^{\star} p<0.05,{ }^{\star \star} /{ }^{\# \#} p<0.01,{ }^{\star \star \star} p<0.001,{ }^{\star \star \star \star} /{ }^{\# \# \# \# p<0.0001 . ~ T w o-t a i l e d ~ S t u d e n t ' s ~ T-t e s t ~}(\mathbf{a}, \mathbf{c}, \mathbf{d}$ bar graphs). Two-way ANOVA (b). Source data are provided as a Source Data file.

were obtained when these precipitations were performed with purified recombinant proteins, we concluded that these interactions were direct (Fig. 7d, e). Furthermore, we found in cotransfection experiments that p62 and NBR1 influence each other's interaction with PPAR $\gamma$ (Supplementary Fig. 5d). Consistently, mutations of p62 (K7A) and NBR1 (D50R) within their respective $\mathrm{PB} 1$ domains that abolished p62-NBR1 interaction ${ }^{11,19,20}$ also clearly abrogated the synergistic binding of p62 and NBR1 to PPAR $\gamma$ (Fig. 7f and Supplementary Fig. 5e). These results demonstrate a nuclear interaction among p62, NBR1, and PPAR $\gamma$, which might influence PPAR $\gamma$ 's activities in BAs.

p62 and NBR1 regulate PPAR $\gamma$-RXRa heterodimerization to control thermogenesis in brown adipocytes. PPAR $\gamma$ functions as an obligate heterodimer with RXRa, which together bind to PPAR-responsive regulatory elements (PPRE) to activate the expression of target genes ${ }^{25}$. Our data also showed that NBR1 coimmunoprecipitated with RXRa in PPAR $y$ immunoprecipitates (Fig. 7c and Supplementary Fig. 5c). Given the functional importance of the PPAR $\gamma$-RXR $\alpha$ interaction, we hypothesized that p62 and NBR1 might play a critical role on its formation. Interestingly, the expression of NBR1 impaired the PPAR $\gamma$-RXR $\alpha$ complex, which was restored by p62 expression (Fig. 8a). However, such a restoration was abolished by mutations that disrupt the interaction between p62 and NBR1 (Fig. 8a). Consistent with this model, the levels of PPAR $\gamma: \mathrm{RXR} \alpha$ heterodimer in coldexposed BAT were reduced in Sqstm1 $1^{-/-}$mice but were normal in Sqstm $1^{-/-} \mathrm{Nbr1}^{-/-}$mice (Fig. 8b). We next reconstituted Sqstm1 and Nbr1 double KO iBAs with either p62 or NBR1 or both together and determined the PPAR $\gamma: \mathrm{RXR} a$ heterodimerization. While p62 was able to enhance interaction between PPAR $\gamma$ and RXRa, NBR1 blunted p62 effects (Fig. 8c). Of great functional relevance, the luciferase assay using a reporter construct revealed an enhanced PPAR $\gamma$ transcriptional activity by p62, but this was reverted by NBR1 co-reconstitution (Fig. 8d). Furthermore, the expression of $U c p 1$, whose transcription is driven by $\operatorname{PPAR} \gamma^{26}$, was increased upon $\mathrm{p} 62$ reconstitution, but the expression of NBR1 severely inhibited p62 effect (Fig. 8e). Our data support the role of p62 and NBR1 in the regulation of PPAR $\gamma: R X R a$ heterodimerization and PPAR $\gamma$-mediated thermogenic program in BAs.

\section{Discussion}

We reported previously that the obese and insulin-resistant phenotypes observed in the whole-body p62 deficiency resulted from reduced systemic $\mathrm{EE}^{14}$ that underscores the critical role of adipocyte's p62 in sustaining $\beta 3$-adrenergic signaling-induced mitochondrial function and thermogenesis in BAT $^{16}$, as well as cancer-associated browning of subcutaneous $\mathrm{WAT}^{17}$. Importantly, the impaired thermogenesis is not secondary to the obese phenotype because impaired EE has previously been demonstrated in newborn p62 mutant pups and in young p62 mutant mice that yet do not differ in body weight or body composition ${ }^{18}$. Further evidence has been shown in isolated and in vitro cultured brown and inguinal white adipocytes harvested from either young lean non-obese p62 mutant mice ${ }^{16,18}$ or neonates from this study.

The severe adiposity and gain of fat mass characteristic of mature p62 mutant mice raises an important question whether glucose tolerance test should be assessed by dosing glucose by lean body mass but not by total body weight. Although dosing according to lean mass has been suggested by some studies especially when body composition is relatively similar, a larger body of literature argues against that because non-lean tissue mass like the white and brown fat and the brain can significantly contribute to whole-body glucose uptake ${ }^{27-29}$. This is particularly 
a

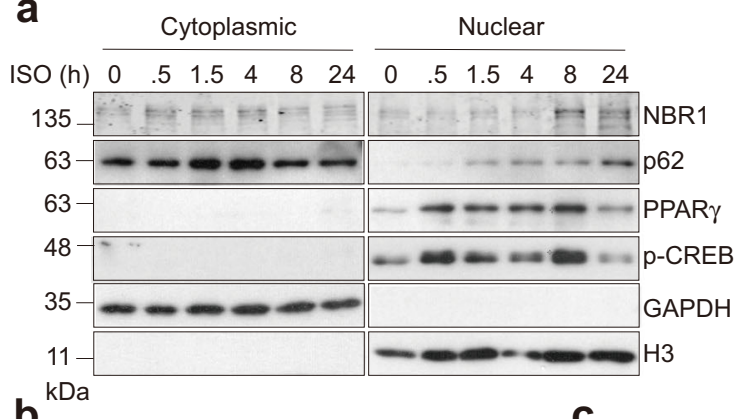

b
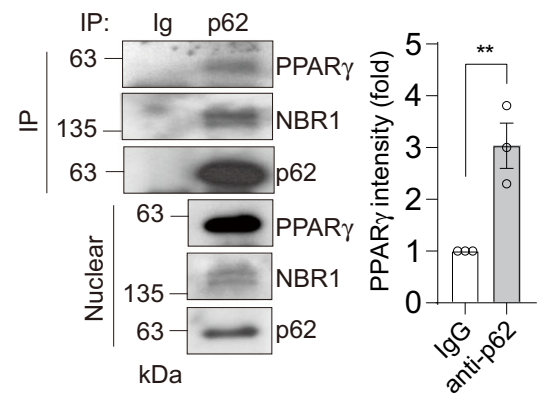

e
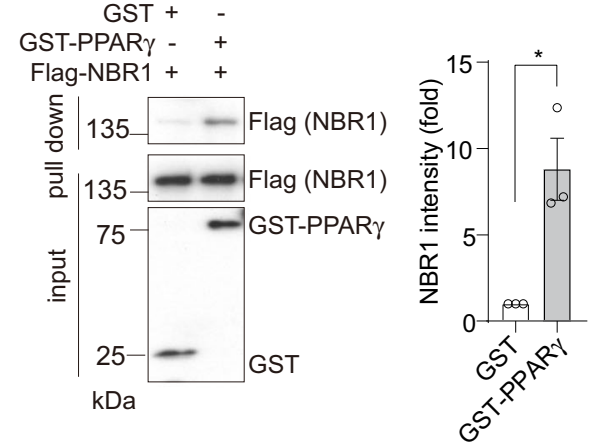

IP: Ig PPAR $\gamma$

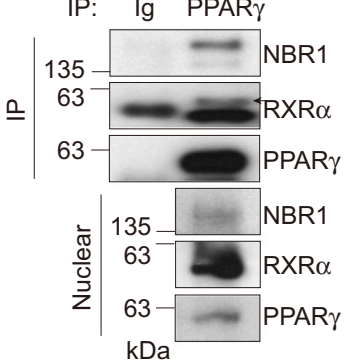

f
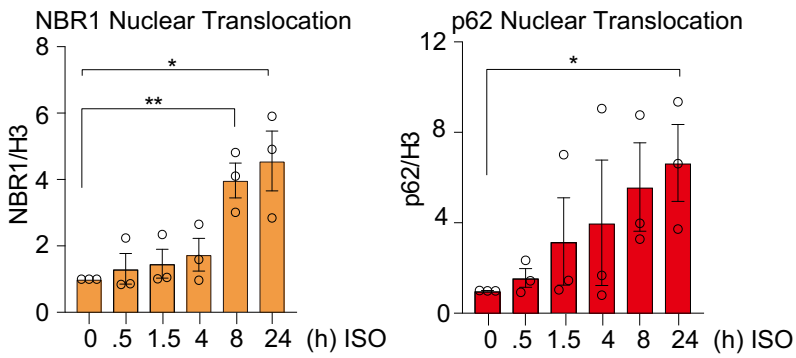

d
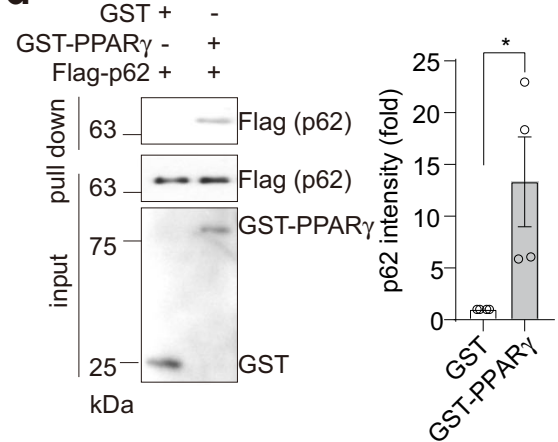

HA-p62 wt + + - +

HA-p62 K7A - - - - +

HA-NBR1 wt +-++

HA-NBR1 D50R - - - +

GST-PPAR $\gamma-++++$

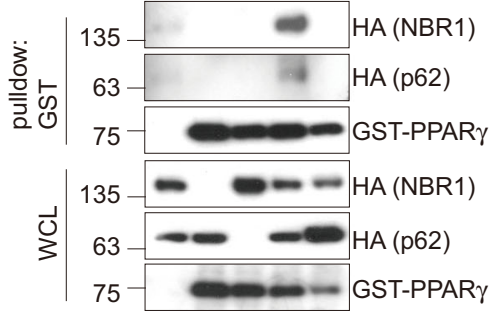

$\mathrm{kDa}$

Fig. 7 p62 and NBR1 interact with PPAR $\boldsymbol{\gamma}$ in brown adipocytes. a Representative immunoblotting of p62 and NBR1 levels in cytoplasmic/nuclear fractions in primary brown adipocytes. Cells were treated with $0.5 \mu \mathrm{M}$ isoproterenol (ISO) for indicated time. Densitometric quantification of nuclear NBR1 and p62 levels was also shown ( $n=3$ independent experiments). b, c Endogenous interaction of PPAR $\gamma$ with p62 and NBR1. p62 (b) or PPAR $\gamma$ (c)

immunoprecipitates from nuclear lysates extracted from ISO and rosiglitazone-treated brown adipocytes were analyzed for the levels of specified proteins. Representative blots and densitometric quantification were shown ( $n=3$ independent experiments for both). d, e Recombinant FLAG-p62 (d) or FLAGNBR1 (e) was incubated with GST and GST-PPAR $\gamma$ proteins separately and the interactions were analyzed by immunoblotting in glutathione-beads pulldown. Representative blots and densitometric quantification were shown ( $n=4$ independent experiments for $\mathbf{d}$ and $n=3$ independent experiments for $\mathbf{e}$ ). f HEK293T cells were transfected with cDNA vectors expressing WT/mutants of HA-p62 or HA-NBR1, and GST-PPAR $\gamma$. The interacting proteins were pulled down using glutathione-beads against GST-PPAR $\gamma$ and analyzed by immunoblotting. Representative blots from three independent replicates with similar results were shown. Data are presented as mean \pm SEM (a-e). ${ }^{\star} p<0.05,{ }^{\star \star} p<0.01$. Two-tailed Student's $T$-test (a-e). Source data are provided as a Source Data file.

important in obese animals, in which the non-lean mass can make up to $50 \%$ of the body weight, and relative to the muscles, adipose glucose uptake is as high as $30 \%$ in obese mice ${ }^{28}$. Thus, total body weight has been adapted for glucose/insulin tolerance tests and normalization of EE data in this study.

Despite the marked phenotypes, the mechanisms whereby p62 deficiency in adipocytes impaired these processes was unclear from those previous studies. The data shown now demonstrate that whole-body and adipocyte-specific NBR1 ablation reverts the obese phenotype induced by 62 deficiency by restoring global EE and thermogenesis in BAT. We also establish here that these in vivo observations stem from a cell autonomous mechanism by which p62 antagonizes an unexpected nuclear function of NBR1 in PPAR $\gamma$ repression. NBR1 shares a remarkable structural similarity with p62 and can physically interact with p62 through their respective $\mathrm{PB} 1$ domains ${ }^{13,19}$. However, the physiological relevance of that interaction was not clear until now.

The PPARs are members of the nuclear receptor (NR) superfamily of ligand-inducible transcription factors. PPRA $\gamma$ is a critical transcriptional regulator of both WAT and BAT development as well as browning of $\mathrm{WAT}^{30}$. Chronic treatment with synthetic ligands of PPAR $\gamma$ strongly induces beige adipocyte differentiation in subcutaneous WAT ${ }^{31}$. PPAR $\gamma$ coordinates with several key co-regulators (PRDM16, PGC1 $\alpha$ ), controlling brown adipogenesis $^{23}$. Ligand binding induces a conformational change in PPRA $\gamma$, promoting dissociation of transcriptional repressors and recruitment of co-activator, leading to activation of downstream gene expression. Collectively, our studies show that upon adrenergic stimulation, PPAR $\gamma$ together with p62 and NBR1 translocates into the nucleus of BAs and establish a multi-protein 
a
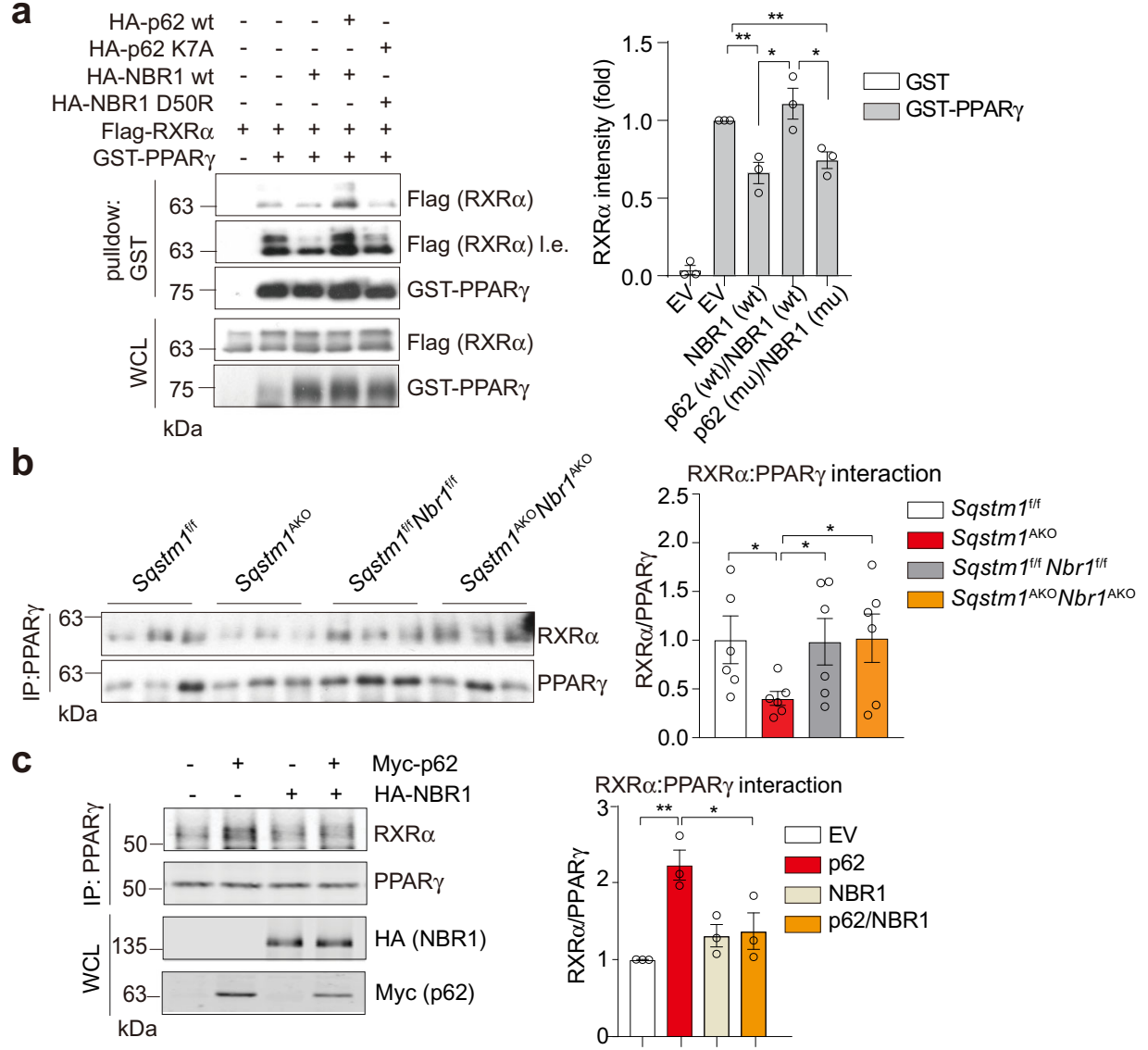

d
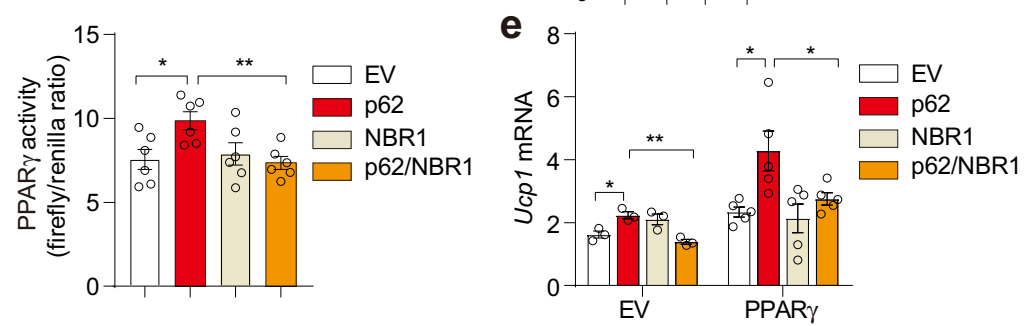

Fig. 8 p62 and NBR1 regulate PPAR $\boldsymbol{\gamma}$-RXR $\alpha$ heterodimerization to control thermogenesis in brown adipocytes. a FLAG-RXR $\alpha, G S T-P P A R \gamma$, WT/ mutants of HA-p62, and HA-NBR1 were overexpressed in HEK293T cells and the interaction of RXR $\alpha$ with PPAR $\gamma$ was analyzed by immunoblotting in pulldowns using glutathione-beads against GST-PPAR $\gamma$, in the present of NBR1 and/or p62. Representative immunoblotting and densitometric quantification were shown ( $n=3$ independent experiments). EV empty vector, wt wild-type, mu mutant. $\mathbf{b}$ Endogenous interaction of PPAR $\gamma$ with RXR $\alpha$ in BAT of mice

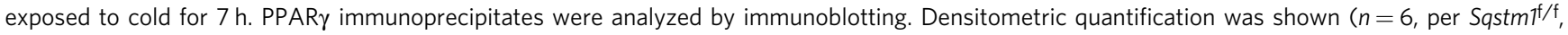

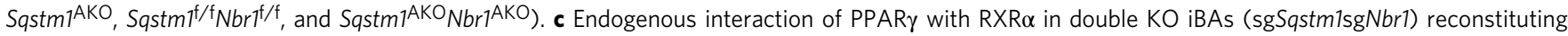
p62, NBR1, or both. PPAR $\gamma$ immunoprecipitates were analyzed by immunoblotting. Densitometric quantification was shown ( $n=3$ independent experiments). $\mathbf{d}$ Luciferase assay determining transcriptional activity of PPAR $\gamma$ in iBAs transfected with indicated cDNA vectors, cells were treated with ISO $(1 \mu \mathrm{M})$ and rosiglitazone $(1 \mu \mathrm{M})$ for $48 \mathrm{~h}$ ( $n=6$ biological replicates). e qPCR analysis of Ucp1 expression in Sqstm1 $1 /-\mathrm{Nbr1} 1^{-/-}$iBAs reconstituting p62 or NBR1 or both with/without overexpression of PPAR $\gamma$. Cells were treated with ISO $(1 \mu M)$ for $48 \mathrm{~h}$. EV $(n=3$ biological replicates), PPAR $(n=5$ biological replicates). Data are presented as mean \pm SEM (a-e). ${ }^{\star} p<0.05,{ }^{\star \star} p<0.01$. Two-tailed Student's $T$-test (a-e). Source data are provided as a Source Data file.

complex. In this way, p62 and NBR1 emerge as co-regulators of PPAR $\gamma$ with opposite activities. That is, whereas p62 favors PPAR $\gamma: R X R a$ heterodimerization to drive thermogenic gene expression, NBR1 impairs PPAR $\gamma: R X R a$ complex formation, decreasing PPAR $\gamma$ activity. Interestingly, our results are consistent with a model whereby p62 contributes to PPAR $\gamma$ activation by restraining NBR1 from its inhibitory binding to PPRA $\gamma$ (Fig. 9). In support of this model, we show here that mutations that disrupt the p62-NBR1 interaction, or depletion of p62, allow the unleashed NBR1 to dampen PPRA $\gamma: R X R a$ heterodimerization and subsequent function.
This proposed mechanism is reminiscent of the role that p62 plays in hepatic stellate cells, in which p62 facilitates the formation of a VDR:RXRa heterodimeric complex through its binding to these $\mathrm{NRs}^{32}$. Thus, it is conceivable that p62 could bridge different NRs, potentially increasing their proximity to respective co-regulators for an optimal transcriptional activation. The ability of p62 to interact with NRs should be considered in the context of a more general role of p62 in the nucleus. In this regard, p62 has also been shown to directly bind ATF4 and to modulate its stability in stromal fibroblasts, which is central to the ATF4mediated metabolic reprogramming of cancer-associated 


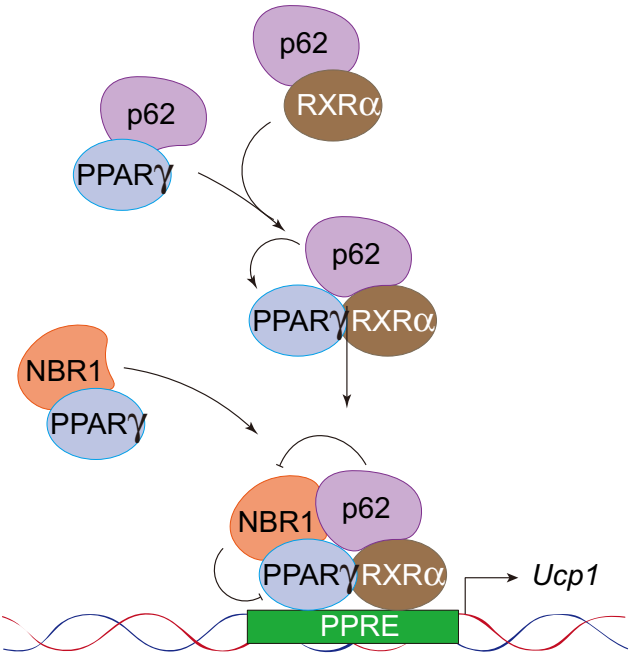

Fig. 9 Model for the role of p62 and NBR1 in the regulation of PPAR $\gamma$ : RXR $\alpha$ heterodimerization and PPAR $\gamma$-mediated thermogenic program in brown adipocytes. $\mathrm{p} 62$ and NBR1 translocate into the nucleus of brown adipocytes upon adrenergic stimulation and form a multi-protein complex with the nuclear receptor PPAR $\gamma$ and its obligate co-activator RXR $\alpha$. p62 separately interacts with PPAR $\gamma$ and RXR $\alpha$, increasing their nuclear proximity and facilitates their heterodimerization to drive thermogenic gene expression. NBR1 emerges as an opposite player that impairs PPAR $\gamma: R X R \alpha$ complex formation and decreases PPAR $\gamma$ activity through direct interaction with PPAR $\gamma$. The fact that p62 and NBR1 synergistically bind to PPAR $\gamma$ allows p62 to restrain NBR1 from its inhibitory binding to PPARy. Nuclear p62 serves as a thermogenic promoter contributing to PPAR $\gamma$ activation in brown adipocytes.

fibroblasts to control tumorigenesis ${ }^{33}$. Direct interaction of p62 with ATF2 is required for genomic binding of ATF2 and ATF2mediated transcription of thermogenic target genes during $\beta$-adrenergic stimulation in $\mathrm{BAs}{ }^{18}$. Whether NBR1 also impacts the VDR, ATF4, and/or the ATF2 systems still needs to be addressed.

Since autophagy is suppressed by $\beta$-adrenergic signaling during fat browning ${ }^{34}$, it is conceivable that the accumulation of $\mathrm{p} 62$ and NBR1 that we describe in this paper could be the consequence of autophagy inhibition. Therefore, autophagy in this context would be a mechanism of fine tuning the signaling capabilities of p62/ NBR1 in thermogenesis and adiposity. Although the role of p62 and NBR1 in mitophagy has been questioned, at least in some systems $^{35}$, it is still possible that mitophagy inhibition might play a role in the regulation of mitochondrial levels during thermogenesis ${ }^{34}$, whereas the accumulated p62-NBR1 tandem described here will insure that mitochondrial biogenesis and UCP1 expression are efficiently activated transcriptionally. Therefore, the p62-NBR1 complex emerges as a central hub organizing two branches of the thermogenic program converging into mitochondrial function, acting both as autophagy adaptors and as signaling mediators through PPAR $\gamma: \mathrm{RXR} \alpha$ transcriptional signaling.

\section{Methods}

Animal. WT and Sqstm1 $1^{-/}$mice were previously described ${ }^{14}$. aP2 cre Sqstm1 flox $\left(\right.$ Sqstm1 $\left.{ }^{\mathrm{AKO}}\right)$ mice were previously described ${ }^{16}$. Nbrl flox mice were available from the previous study ${ }^{20}$. Sqstm 1 and $\mathrm{Nbr} 1$ dual flox mice were generated by cross-bred with individual flox mice than to breed to aP2 cre to generate adipocyte-specific $\mathrm{KO}$ mice (Sqstm $1^{\mathrm{AKO}} \mathrm{Nbr} 1^{\mathrm{AKO}}$ mice). All mouse strains were generated in a C57BL/6 background. All mice were born and maintained under pathogen-free conditions. Mice were fed a normal chow diet and kept on a 12-h light/12-h dark cycle with free access to food and water in a temperature $\left(22 \pm 1^{\circ} \mathrm{C}\right)$ and humidity $(50 \pm 5 \%)$ controlled room. All genotyping was done by PCR. Mice were sacrificed, and adipose tissues and liver sections were dissected. Animal handling and experimental procedures conformed to institutional guidelines and were approved by the Sanford Burnham Prebys Medical Discovery Institute Institutional Animal Care and Use Committee.

Metabolic phenotyping. EE, $\mathrm{O}_{2}$ consumption rate, $\mathrm{CO}_{2}$ production rate, RER, food intake, and locomotor activity were assessed in male mice at 50-55 weeks of age using an automated indirect calorimetry Oxymax system of the Comprehensive Lab Animal Monitoring System (CLAMS; Promethium System) at UCSD. After $48 \mathrm{~h}$ of adaptation, $\mathrm{O}_{2}$ consumption and $\mathrm{CO}_{2}$ production were measured to determine the respiratory quotient and EE. EE was analyzed using ANCOVA with body weight as covariate as previously described ${ }^{22,36}$. Whole-body composition (fat and lean mass) was measured using Dual-Energy X-ray Absorptiometry at UCSD Animal Care Program. For glucose tolerance test, mice at 25-28 weeks of age were fasted overnight and then challenged with $1.5 \mathrm{~g}$ glucose per $\mathrm{kg}$ body weight. For insulin tolerance test, mice at 25-28 weeks of age were fasted $4 \mathrm{~h}$ and administrated with $0.5 \mathrm{U}$ insulin per $\mathrm{kg}$ body weight. Glucose concentrations of tail blood were then measured by using an ACCU-CHEK Aviva (Roche) glucometer at indicated time points. For acute cold exposure, all aP2 Cre mouse lines at 25-27 weeks of age were singly housed at $4{ }^{\circ} \mathrm{C}$ in a non-bedded cage with access to water but not food for $7 \mathrm{~h}$. Core body temperature was measured using a rectal probe (BAT-10, Physitemp). At the end of the experiment, BAT was resected for histological and gene expression analyses. To induce browning in WAT, mice at 25-27 weeks of age were i.p. administrated with $\beta 3$-adrenergic agonist CL316,243 at $0.5 \mathrm{mg} / \mathrm{kg}$ BW for 4 days and at $1 \mathrm{mg} / \mathrm{kg}$ for the last day. After injection for 5 days, inguinal WAT was dissected for histological and gene expression analyses.

Histological analysis. Tissues from indicated mice were isolated, rinsed in ice-cold PBS, fixed in $10 \%$ neutral buffered formalin for $24 \mathrm{~h}$, dehydrated, and embedded in paraffin. Livers were embedded in Tissue Tek O.C.T. compound and snap frozen in dry ice, then kept in $-80^{\circ} \mathrm{C}$. Tissue sections $(5 \mu \mathrm{m})$ were stained with hematoxylin and eosin (H\&E). Histological sections of fat pads were stained with $\mathrm{H} \& \mathrm{E}$ and captured under 20-fold magnification by the AxioVision LE software to determine adipocyte size. At least seven fields per section from four different mice of each genotype were randomly selected to determine the adipocyte size and number according to morphological feature using "ImageJ"-based software "Adiposoft." Frozen liver sections $(5 \mu \mathrm{m})$ were stained with ORO (Sigma-Aldrich) to detect lipid accumulation. Sections were fixed in paraformaldehyde and stained for $3 \mathrm{~h}$ in $0.5 \%$ ORO in propylene glycol, followed by $1 \mathrm{~min}$ incubations in $85 \%$ aqueous propylene glycol. After the slides were washed in distilled water, they were counterstained with Harris's hematoxylin for $10 \mathrm{~s}$.

Lipid analysis. For determination of lipids mass, liver sample were washed with PBS and frozen. Total lipids were isolated from homogenates by Folch extraction. Briefly, around $50 \mathrm{mg}$ tissue samples were homogenized in $1 \mathrm{ml}$ methanol, homogenates were further mixed with $2 \mathrm{ml}$ chloroform and rotated mildly for $2 \mathrm{~h}$ to extract lipid. Samples were then mixed roughly with $1 \mathrm{ml} \mathrm{H}_{2} \mathrm{O}$ for $30 \mathrm{~s}$ to separate phases. The lipid-containing organic phase (bottom) was collected and dried by nitrogen. Total lipids were dissolved in PBS containing $1 \%$ Triton X-100, followed by quantification by kits. The tissue lipid concentrations were determined spectrophotometrically (Wako Diagnostics, USA) and normalized to tissue weight.

Cell culture. HEK293T/HEK293FT cells were purchased from ATCC. Primary and iBAs were generated in house. HEK293T/HEK293FT cells and iBAs were cultured in Dulbecco's Modified Eagles Medium (DMEM, Corning) supplemented with 10\% fetal bovine serum (FBS), $2 \mathrm{mM}$ glutamine, and primary cells were cultured in DMEM/F-12 supplemented with GlutaMAX (Gibco) and 10\% FBS. All cells were maintained in an atmosphere of $95 \%$ air and $5 \% \mathrm{CO}_{2}$. Only cells that were tested negative for mycoplasm were used for experiments.

Generation of primary and immortalized brown adipocytes. For preparation of primary BA, BAT was excised from neonates of WT, Sqstm1 $1^{-/-}, \mathrm{Nbr1}^{-/-}$, and Sqstm $1^{-/-} \mathrm{Nbr1}^{-/-}$mice regardless of gender, and minced in $2 \mathrm{ml} \mathrm{PBS}$, then added with collagenase $\mathrm{D}(1.5 \mathrm{U} / \mathrm{ml})$, dispase II $(2.4 \mathrm{U} / \mathrm{ml})$, and $\mathrm{CaCl}_{2}(10 \mathrm{mM})$ and the tissues were incubated at $37^{\circ} \mathrm{C}$ with gentle shaking $(30 \mathrm{~min})$. Larger particles were removed using a $100 \mu \mathrm{m}$ cell strainer, and the filtrates were centrifuged at $500 \mathrm{~g}$ for 5 min three times to pellet SVF and remove collagenase residue. Isolated SVF were seeded in culture dish for 4 days to eliminate the unattached dead cell/white blood cell populations. SVF including adipogenic precursors were passed into 6-well plate for in vitro differentiation. Two days post confluence, differentiation was initiated by induction cocktail (DMEM/F-12 containing 10\% FBS, $2 \mu \mathrm{g} / \mathrm{ml}$ dexamethasone, $0.5 \mathrm{mM}$ isobutylmethylxanthine, $125 \mu \mathrm{M}$ indomethacine, $1 \mathrm{nM} \mathrm{T}, 0.5 \mu \mathrm{g} / \mathrm{ml}$ insulin, and $1 \mu \mathrm{M}$ rosiglitazone) for 2 days, followed by maintenance cocktail (DMEM/F-12 containing $1 \mathrm{nM} \mathrm{T3}, 0.5 \mu \mathrm{g} / \mathrm{ml}$ insulin, and $1 \mu \mathrm{M}$ rosiglitazone and $10 \%$ FBS) for another 5 days to become fully differentiated. To generate iBAs, BAT SVF were immortalized by retroviral pBabe-zeo-LT-ST (SV40) and selected by Zeocin. Single cell clone was selected and tested for differentiation capacity. All selected clones used in this study maintain efficient differentiation potential and express the BA marker UCP1. Immortalized SVF are cultured in DMEM supplemented with $10 \%$ FBS (without pyruvate). For differentiation of iBAs, the 
induction cocktail contains dexamethasone $(2 \mu \mathrm{g} / \mathrm{ml})$, isobutylmethylxanthine $(0.5 \mathrm{mM})$, indomethacine $(125 \mu \mathrm{M})$, rosiglitazone $(0.5 \mu \mathrm{M}), \mathrm{T} 3(1 \mathrm{nM})$, and insulin $(5 \mu \mathrm{g} / \mathrm{ml})$ in growth medium. After 2 days, cells were maintained in growth medium supplemented only with rosiglitazone, T3, and insulin till day 7 for experiments. For transfection assay, differentiating cells at day 5 were transfected with indicated vectors using X-tremeGENE HP transfection reagent (Roche). To stimulate thermogenesis, cells were treated with ISO (Sigma-Aldrich) for indicated times when cells were fully differentiated.

Generation of knockout cell by CRISPR/Cas9. To knockout p62 and NBR1 in immortalized SVF cells, 20-nucleotide single-guide RNA sequences targeting the mouse genes (GACUCUCCCUGCAGAGAAGA for Sqstm1 and CUACAGA UGCAAGUCCACGA for Nbr1) were purchased from Synthego and transduced into cells with recombinant Streptococcus pyogenes Cas9 protein (Truecut Cas9 Protein v2, Thermo) using Neon Electroporation System (Invitrogen). Single clones were expanded and screened for p62 and NBR1 expression by protein immunoblotting.

Cytoplasmic and nuclear fractionation. Adipocytes were differentiated in P100 dishes. At day 7, mature adipocytes were lysed on ice with Buffer A (20 mM Tris$\mathrm{HCl}$ at $\mathrm{pH} 7.9,1.5 \mathrm{mM} \mathrm{MgCl}_{2}, 10 \mathrm{mM} \mathrm{KCl}$ ) with phosphatase and proteinase inhibitors. Lysates were centrifuged at $750 \mathrm{~g}$ for $5 \mathrm{~min}$ at $4{ }^{\circ} \mathrm{C}$. The supernatant was collected and centrifuged at $9000 \mathrm{~g}$ for $10 \mathrm{~min}$ (cytoplasmic fraction). The pellets from previous centrifuge were washed by Buffer $\mathrm{A}$ to remove cytoplasmic contamination, then resuspended in Buffer C (20 mM Tris- $\mathrm{HCl}$ at pH7.9, $1.5 \mathrm{mM}$ $\mathrm{MgCl}_{2}, 0.42 \mathrm{M} \mathrm{NaCl}$ ) followed by sonication. Lysates were centrifuged at $16,800 \mathrm{~g}$ for $15 \mathrm{~min}$ and the resulting supernatant was collected as nuclear fraction.

Immunoprecipitation. For total cell lysis, cells were rinsed once with ice-cold PBS and lysed on mice in $\sigma 3$ lysis buffer $(25 \mathrm{mM}$ Tris- $\mathrm{HCl}$ at $\mathrm{pH} 8.0,100 \mathrm{mM} \mathrm{NaCl}, 1 \%$ Triton X-100, 10\% glycerol) with phosphatase and proteinase inhibitors. Lysates were centrifuged at $13,000 \mathrm{~g}$ for $15 \mathrm{~min}$ at $4{ }^{\circ} \mathrm{C}$ to remove cell debris. For tissue extracts, BAT was homogenized in $0.5 \mathrm{ml}$ ice-cold lysis buffer $(50 \mathrm{mM}$ HEPES at pH7.5, $150 \mathrm{mM} \mathrm{NaCl}, 2.5 \mathrm{mM}$ EDTA, $2.5 \mathrm{mM}$ EGTA, $1 \%$ NP-40. $10 \%$ glycerol). Homogenates were centrifuged at $13,000 \mathrm{~g}$ for $15 \mathrm{~min}$ at $4{ }^{\circ} \mathrm{C}$ to remove fat layer and insoluble material. Protein content of lysates was quantified by $D C$ Protein Assay Kit (Bio-Rad). For immunoprecipitation, $1 \mathrm{mg}$ proteins of tissue or total cell lysates, 3-4 mg proteins of nuclear extracts from cells (for immunoprecipitating endogenous protein), or $0.5 \mathrm{mg}$ of total cell lysates (for co-transfection immunoprecipitation) were pre-cleared with $30 \mu \mathrm{l} 50 \%$ slurry of protein $\mathrm{G}$ agarose (Genesee Scientific) for $30 \mathrm{~min}$. Then $2 \mu \mathrm{g}$ of primary antibodies (anti-p62, Progen, \#GP62C; anti-PPAR $\gamma$, Santa Cruz Biotechnology, \#sc-7273; anti-FLAG, Sigma-Aldrich, \#P2983) or control immunoglobulins were added to the lysates and incubated with rotation overnight at $4{ }^{\circ} \mathrm{C}$. The next day, $30 \mu \mathrm{l} 50 \%$ slurry of protein $\mathrm{G}$ was added to the extracts for additional $1 \mathrm{~h}$. Immunoprecipitates were obtained by centrifuge at $2000 \mathrm{~g}$ at $4{ }^{\circ} \mathrm{C}$ for $5 \mathrm{~min}$, followed by washing several times with BC300 buffer (50 mM Tris- $\mathrm{HCl}$ at pH7.9, $300 \mathrm{mM} \mathrm{KCl,} 2 \mathrm{mM}$ EDTA, $10 \%$ glycerol, $0.1 \% \mathrm{NP}-40$ ) for nuclear extract, or the same lysis buffers for total cell lysates and tissue lysates. Immunoprecipitated proteins were denatured by adding $10 \mu \mathrm{l}$ of sample buffer and boiled for $10 \mathrm{~min}$ before subjection to immunoblotting.

GST pull-down assay. For the purification of Flag-p62 and Flag-NBR1 proteins, HEK293T cells in P150 dishes were transfected with $20 \mu \mathrm{g}$ cDNA vectors of each genes. After $48 \mathrm{~h}$, cells were lysed in lysis buffer $(50 \mathrm{mM}$ Tris- $\mathrm{HCl}$ at $\mathrm{pH} 7.4$, $150 \mathrm{mM} \mathrm{NaCl}, 1 \mathrm{mM}$ EDTA, $1 \%$ Triton X-100) with phosphatase and protease inhibitors on ice for $30 \mathrm{~min}$. After centrifugation at $13,000 \mathrm{~g}$ at $4{ }^{\circ} \mathrm{C}$ for $15 \mathrm{~min}$, cell lysates were added with $20 \mu \mathrm{l}$ slurry of Anti-FLAG M2 affinity gel (Sigma-Aldrich) for $2 \mathrm{~h}$. Then, immunoprecipitates were centrifuged at $2000 \mathrm{~g}$ at $4{ }^{\circ} \mathrm{C}$ for $5 \mathrm{~min}$ and washed with TBS buffer ( $50 \mathrm{mM}$ Tris- $\mathrm{HCl}$ at $\mathrm{pH} 7.4,150 \mathrm{mM} \mathrm{NaCl}$ ) three times to remove unspecific binding. FLAG-tagged proteins were eluted by 3XFLAG peptide (Sigma-Aldrich) at $100 \mu \mathrm{g} / \mathrm{ml}$ for $30 \mathrm{~min}$ at $4{ }^{\circ} \mathrm{C}$ in gentle shaking. Eluted proteins were aliquoted and stored in $-80^{\circ} \mathrm{C}$ immediately. To generate GST, GST-fused PPAR $\gamma$ proteins, HEK293T cells in P100 dishes were transfected with $10 \mu \mathrm{g}$ cDNA vectors of each genes. After $48 \mathrm{~h}$, cells were lysed on ice for $30 \mathrm{~min}$ in RIPA lysis buffer with phosphatase and protease inhibitors. After centrifugation at 13,000 g at $4{ }^{\circ} \mathrm{C}$ for $15 \mathrm{~min}$, cell lysates were added with $30 \mu \mathrm{l} 50 \%$ slurry of glutathione agarose (Thermo Scientific) for $4 \mathrm{~h}$ followed by washing with RIPA buffer for four times. For in vitro pull-down assay, $20 \mu \mathrm{l}$ of glutathione agarose-bound GST-PPAR $\gamma$, GST were mixed with $1 \mu \mathrm{l}$ of eluted FLAG-p62 or FLAG-NBR1 in $500 \mu$ l of NETE-N binding buffer (50 mM Tris- $\mathrm{HCl}$ at $\mathrm{pH} 8.0,100 \mathrm{mM} \mathrm{NaCl}, 6 \mathrm{mM}$ EDTA, $0.5 \%$ NP40) with phosphatase and protease inhibitors for $1 \mathrm{~h}$. After that, samples were washed several times with $1 \mathrm{ml}$ of binding buffer and proteins were denatured by adding $20 \mu \mathrm{l}$ of sample buffer followed by boiling for $10 \mathrm{~min}$, subjected to immunoblotting. For the co-transfection pull-down assay, HEK293T cells in P60 dishes were transfected with $4 \mu \mathrm{g}$ cDNA vectors of HA-tagged p62 or NBR1, FLAG-tagged RXRa, and GST-tagged PPAR $\gamma$. After $48 \mathrm{~h}$, cells were lysed on ice for $30 \mathrm{~min}$ in lysis buffer (50 mM HEPES at pH7.5, $150 \mathrm{mM} \mathrm{NaCl}, 2.5 \mathrm{mM}$ EDTA, 2.5 mM EGTA, $1 \%$ NP- $40.10 \%$ glycerol) with phosphatase and protease inhibitors. After centrifugation at $13,000 \mathrm{~g}$ at $4{ }^{\circ} \mathrm{C}$ for $15 \mathrm{~min}$, cell lysates were added with
$30 \mu \mathrm{l} 50 \%$ slurry of glutathione agarose beads overnight followed by washing with lysis buffer for four times. Proteins pulled down by GST-PPAR $\gamma$ were denatured by adding $40 \mu \mathrm{l}$ of sample buffer followed by boiling for $10 \mathrm{~min}$, subjected to immunoblotting.

Immunoblot analysis. Protein extracts were separated by SDS-PAGE and transferred to Immobilon-P PVDF membranes (Millipore). After blocking with 5\% nonfat dry milk in Tris-buffered saline and $0.1 \%$ Tween, the membranes were incubated overnight at $4{ }^{\circ} \mathrm{C}$ with the indicated antibodies. The following antibodies were used: GAPDH (Santa Cruz Biotech; \#sc-32233; Dilution: 1:20,000), $\beta$-actin (Sigma-Aldrich; \#A1978; Dilution 1:20,000), H3 (Abcam; \#ab1791; Dilution 1:25,000), UCP1 (Abcam; \#ab10983; Dilution: 1:6,000), alpha Tubulin (Santa Cruz Biotech; \#sc-8035; Dilution: 1:1000), phospho-CREB (Ser133) (Cell signaling; \#9198; Dilution 1:1,000), PPAR (Santa Cruz Biotech; \#sc-7273; Dilution 1:800), PPAR $\gamma$ (Cell signaling; \#2443; Dilution 1:1,000), RXRa (Santa Cruz Biotech; \#sc553; Dilution 1:500), RXRa/ $/ \gamma$ (Santa Cruz Biotech; \#sc-774; Dilution 1:500), p62 (Rodent Specific) (Cell signaling; \#23214; Dilution 1:1000), p62, (Progen; \#GP62-C; Dilution 1:1000), p62 (Thermo Scientific; \#PA5-20839; Dilution 1:1000), NBR1 (Santa Cruz Biotech; \#sc-130380; Dilution 1:800), SDHB (Santa Cruz Biotech; \#sc271548; Dilution 1:2,000), UQCRC2 (Santa Cruz Biotech; \#sc-390378; Dilution 1:2,000), GST (Santa Cruz Biotech; \#sc-138; Dilution 1:500), HA (Santa Cruz Biotech; \#sc-7392; Dilution 1:500), FLAG (Sigma-Aldrich; \#F1804; Dilution 1:4,000), Myc (Santa Cruz Biotech; \#sc-40; Dilution 1:500). After $1 \mathrm{~h}$ incubation with the appropriate secondary horseradish peroxidase-conjugated antibodies including anti-mouse IgG1(BD Biosciences; \#550331; Dilution 1:3,000) and antirabbit IgG (Dako; \#E0432; Dilution 1:3,000), the immune complexes were detected by chemiluminescence (Thermo Scientific). The gels/blots with the same experiment were processed in parallel for optimal quantitative comparisons. Densitometric quantification of protein intensity from western blot was assessed by ImageJ software. Images of uncropped blots are provided in the Source Data file.

Luciferase assay. PPAR transcriptional activity was monitored in vitro using a reporter construct consisting of three PPRE copies upstream of a luciferase reporter. At day 5 of differentiation, BAs differentiated from immortalized SVF were transiently transfected with the following plasmids using X-tremeGENE: PPREx3-TK-luc (Addgene\#1015), pRL-TK (control Renilla), HA-p62, HA-NBR1 or Flag-PPAR $\gamma 1$ (Addgene\#78769). The level of promoter activity was evaluated by determining the firefly luciferase activity relative to renilla luciferase activity using the Dual Luciferase Assay System (Promega) according to the manufacturer's instruction.

RNA analysis. Total RNA from mouse tissues and cultured cells was isolated using the TRIZOL reagent (Invitrogen) and the RNeasy Mini Kit (QIAGEN), followed by DNase treatment. After quantification using a Nanodrop 1000 spectrophotometer (Thermo Scientific), RNA was reverse transcribed using random primers and MultiScribe Reverse Transcriptase (Applied Biosystems). Gene expression was analyzed by amplifying $20 \mathrm{ng}$ of the complementary DNA using the CFX96 Real Time PCR Detection System with SYBR Green Master Mix (Bio-Rad). The amplification parameters were set at $95^{\circ} \mathrm{C}$ for $30 \mathrm{~s}, 58^{\circ} \mathrm{C}$ for $30 \mathrm{~s}$, and $72^{\circ} \mathrm{C}$ for $30 \mathrm{~s}$ ( 40 cycles total). Gene expression values for each sample were normalized to the 18S RNA. A complete list of all primers used is listed in Supplementary Table 1.

Measurement of respiration in adipocytes. The cellular OC of BAs was determined using an XFp Extracellular Flux Analyzer and analyzed by Agilent Seahorse Wave Software (Seahorse Bioscience). Prior to assay, 10,000 immortalized SVF cells were seeded into XFp microplates. One day later, Adipogenic differentiation was initiated using a protocol mentioned above. Seven days post differentiation, adipocyte culture medium was changed to XF basal medium containing $5 \mathrm{mM}$ glucose, $1 \mathrm{mM}$ pyruvate, and $2 \mathrm{mM}$ GlutaMAX. The basal uncoupled OCR was determined using $1 \mu \mathrm{M}$ oligomycin. To determine the impact of ISO stimulation in uncoupled OCR. Then, $5 \mu \mathrm{M}$ ISO was injected three cycles after oligomycin injection. Oxygen consumption values were normalized to protein content.

Statistical analysis. GraphPad Prism software (v. 8.3.0) was used for graphing and statistical analysis. For comparison between two groups, datasets were analyzed by Unpaired Student's two-tailed $T$-test. Multiple comparisons were analyzed by twoway ANOVA to determine the statistical significance between groups on the basis of one variable. Differences in EE were calculated using ANCOVA with body weight as covariate using SPSS (version 24). Values of $p<0.05$ were considered as significantly different.

Reporting summary. Further information on research design is available in the Nature Research Reporting Summary linked to this article.

\section{Data availability}

The data that support the findings of this study are provided in the data source file and available from the corresponding author upon reasonable request. The statistical $p$ value 
from GraphPad Prism or SPSS reports is provided in the individual figure

legends. Source Data are provided with this paper.

Received: 6 April 2020; Accepted: 13 April 2021;

Published online: 17 May 2021

\section{References}

1. Cannon, B. \& Nedergaard, J. Brown adipose tissue: function and physiological significance. Physiol. Rev. 84, 277-359 (2004).

2. Betz, M. J. \& Enerback, S. Targeting thermogenesis in brown fat and muscle to treat obesity and metabolic disease. Nat. Rev. Endocrinol. 14, 77-87 (2018).

3. Cypess, A. M. et al. Identification and importance of brown adipose tissue in adult humans. N. Engl. J. Med. 360, 1509-1517 (2009).

4. van Marken Lichtenbelt, W. D. et al. Cold-activated brown adipose tissue in healthy men. N. Engl. J. Med. 360, 1500-1508 (2009).

5. Saito, M. et al. High incidence of metabolically active brown adipose tissue in healthy adult humans: effects of cold exposure and adiposity. Diabetes 58, 1526-1531 (2009).

6. $\mathrm{Wu}, \mathrm{J}$. et al. Beige adipocytes are a distinct type of thermogenic fat cell in mouse and human. Cell 150, 366-376 (2012).

7. Harms, M. \& Seale, P. Brown and beige fat: development, function and therapeutic potential. Nat. Med. 19, 1252-1263 (2013).

8. Moscat, J. \& Diaz-Meco, M. T. p62 at the crossroads of autophagy, apoptosis, and cancer. Cell 137, 1001-1004 (2009).

9. Moscat, J., Karin, M. \& Diaz-Meco, M. T. p62 in cancer: signaling adaptor beyond autophagy. Cell 167, 606-609 (2016).

10. Duran, A. et al. The signaling adaptor p62 is an important NF-kappaB mediator in tumorigenesis. Cancer Cell 13, 343-354 (2008).

11. Duran, A. et al. p62 is a key regulator of nutrient sensing in the mTORC1 pathway. Mol. Cell 44, 134-146 (2011).

12. Valencia, T. et al. Metabolic reprogramming of stromal fibroblasts through p62-mTORC1 signaling promotes inflammation and tumorigenesis. Cancer Cell 26, 121-135 (2014).

13. Moscat, J. \& Diaz-Meco, M. T. Feedback on fat: p62-mTORC1-autophagy connections. Cell 147, 724-727 (2011).

14. Rodriguez, A. et al. Mature-onset obesity and insulin resistance in mice deficient in the signaling adapter p62. Cell Metab. 3, 211-222 (2006).

15. Lee, S. J. et al. A functional role for the p62-ERK1 axis in the control of energy homeostasis and adipogenesis. EMBO Rep. 11, 226-232 (2010).

16. Muller, T. D. et al. p62 links beta-adrenergic input to mitochondrial function and thermogenesis. J. Clin. Invest. 123, 469-478 (2013).

17. Huang, J. et al. Adipocyte p62/SQSTM1 suppresses tumorigenesis through opposite regulations of metabolism in adipose tissue and tumor. Cancer Cell 33, 770-784 (2018). e776.

18. Fischer, K. et al. The scaffold protein p62 regulates adaptive thermogenesis through ATF2 nuclear target activation. Nat. Commun. 11, 2306 (2020).

19. Moscat, J., Diaz-Meco, M. T., Albert, A. \& Campuzano, S. Cell signaling and function organized by PB1 domain interactions. Mol. Cell 23, 631-640 (2006).

20. Hernandez, E. D. et al. A macrophage NBR1-MEKK3 complex triggers JNKmediated adipose tissue inflammation in obesity. Cell Metab. 20, 499-511 (2014).

21. Weisberg, S. P. et al. Obesity is associated with macrophage accumulation in adipose tissue. J. Clin. Invest. 112, 1796-1808 (2003).

22. Tschop, M. H. et al. A guide to analysis of mouse energy metabolism. Nat. Methods 9, 57-63 (2011).

23. Inagaki, T., Sakai, J. \& Kajimura, S. Transcriptional and epigenetic control of brown and beige adipose cell fate and function. Nat. Rev. Mol. Cell Biol. 17, 480-495 (2016).

24. Basse, A. L. et al. Regulation of glycolysis in brown adipocytes by HIF-1alpha. Sci. Rep. 7, 4052 (2017).

25. Chawla, A., Repa, J. J., Evans, R. M. \& Mangelsdorf, D. J. Nuclear receptors and lipid physiology: opening the X-files. Science 294, 1866-1870 (2001).

26. Cao, W. et al. p38 mitogen-activated protein kinase is the central regulator of cyclic AMP-dependent transcription of the brown fat uncoupling protein 1 gene. Mol. Cell Biol. 24, 3057-3067 (2004).

27. Fam, B. C. et al. Normal muscle glucose uptake in mice deficient in muscle GLUT4. J. Endocrinol. 214, 313-327 (2012).

28. Makarova, E. et al. Decreases in circulating concentrations of long-chain acylcarnitines and free fatty acids during the glucose tolerance test represent tissue-specific insulin sensitivity. Front Endocrinol. (Lausanne) 10, 870 (2019).

29. Higgins, J., Proctor, D. \& Denyer, G. Aging changes tissue-specific glucose metabolism in rats. Metabolism 48, 1445-1449 (1999).
30. Ahmadian, M. et al. PPARgamma signaling and metabolism: the good, the bad and the future. Nat. Med. 19, 557-566 (2013).

31. Ohno, H., Shinoda, K., Spiegelman, B. M. \& Kajimura, S. PPARgamma agonists induce a white-to-brown fat conversion through stabilization of PRDM16 protein. Cell Metab. 15, 395-404 (2012).

32. Duran, A. et al. p62/SQSTM1 by binding to vitamin D receptor inhibits hepatic stellate cell activity, fibrosis, and liver cancer. Cancer Cell 30, 595-609 (2016).

33. Linares, J. F. et al. ATF4-induced metabolic reprograming is a synthetic vulnerability of the p62-deficient tumor stroma. Cell Metab. 26, 817-829.e816 (2017).

34. Altshuler-Keylin, S. et al. Beige adipocyte maintenance is regulated by autophagy-induced mitochondrial clearance. Cell Metab. 24, 402-419 (2016).

35. Lazarou, $\mathrm{M}$. et al. The ubiquitin kinase PINK1 recruits autophagy receptors to induce mitophagy. Nature 524, 309-314 (2015).

36. Speakman, J. R., Fletcher, Q. \& Vaanholt, L. The ' 39 steps': an algorithm for performing statistical analysis of data on energy intake and expenditure. Dis. Model Mech. 6, 293-301 (2013).

\section{Acknowledgements}

The research was supported by grants by NCI and NIDDK of the National Institutes of Health under awards numbers: R01DK108743, R01CA211794 to J. M; R01CA218254 to M. T. D.-M.; T. D. M. received grant support from the German Research Foundation (DFG-TRR152 and DFG-TRR296). The content is solely the responsibility of the authors and does not necessarily represent the official views of the National Institutes of Health. J. M. and M. T. D.-M. are Homer T. Hirst III Professors of Oncology in Pathology. We thank Alyssa Ruelos and the personnel of the Histology, Cell Imaging, Genomics, Viral Vectors, Animal Facility, and Shared Resources at SBP Medical Discovery Institute and the UCSD Animal Care Program for technical assistance.

\section{Author contributions}

J. H., M. T. D.-M., and J. M. designed the experiments. J. H., J. F. L., and W. X. performed the experiments and analyzed the data. A. D. generated mouse lines. T. D. M. provided p $62^{\mathrm{f} / \mathrm{f}} \mathrm{aP} 2-C r e$ mice and expertise in mouse metabolism and analyzed data. A. R. S. offered access to the CLAMS study. All authors discussed the results and commented on the manuscript. M. T. D.-M. and J. M. conceived and supervised the project. J. H., M. T. D.-M., and J. M. wrote the manuscript.

\section{Competing interests}

The authors declare no competing interests.

\section{Additional information}

Supplementary information The online version contains supplementary material available at https://doi.org/10.1038/s41467-021-23085-0.

Correspondence and requests for materials should be addressed to J.M.

Peer review information Nature Communications thanks Alexander Banks, Walter Wahli, and the other, anonymous, reviewer(s) for their contribution to the peer review of this work.

Reprints and permission information is available at http://www.nature.com/reprints

Publisher's note Springer Nature remains neutral with regard to jurisdictional claims in published maps and institutional affiliations.

Open Access This article is licensed under a Creative Commons Attribution 4.0 International License, which permits use, sharing, adaptation, distribution and reproduction in any medium or format, as long as you give appropriate credit to the original author(s) and the source, provide a link to the Creative Commons license, and indicate if changes were made. The images or other third party material in this article are included in the article's Creative Commons license, unless indicated otherwise in a credit line to the material. If material is not included in the article's Creative Commons license and your intended use is not permitted by statutory regulation or exceeds the permitted use, you will need to obtain permission directly from the copyright holder. To view a copy of this license, visit http://creativecommons.org/ licenses/by/4.0/

(C) The Author(s) 2021 